\title{
Conditional Quantum Dynamics with Several Observers
}

\author{
Jacek Dziarmaga ${ }^{1,2}$, Diego A. R. Dalvit ${ }^{1}$ and Wojciech H. Zurek ${ }^{1}$ \\ 1) Los Alamos National Laboratory, Theoretical Division, MS-B213, Los Alamos, New Mexico 87545 \\ 2) Instytut Fizyki Uniwersytetu Jagiellońskiego, ul. Reymonta 4, 30-059 Kraków, Poland
}

\begin{abstract}
We consider several observers who monitor different parts of the environment of a single quantum system and use their data to deduce its state. We derive a set of conditional stochastic master equations that describe the evolution of the density matrices each observer ascribes to the system under the Markov approximation, and show that this problem can be reduced to the case of a single "super-observer", who has access to all the acquired data. The key problem - consistency of the sets of data acquired by different observers - is then reduced to the probability that a given combination of data sets will be ever detected by the "super-observer". The resulting conditional master equations are applied to several physical examples: homodyne detection of phonons in quantum Brownian motion, photo-detection and homodyne detection of resonance fluorescence from a two-level atom. We introduce relative purity to quantify the correlations between the information about the system gathered by different observers from their measurements of the environment. We find that observers gain the most information about the state of the system and they agree the most about it when they measure the environment observables with eigenstates most closely correlated with the optimally predictable pointer basis of the system.
\end{abstract}

PACS numbers: PACS numbers: 03.65.Bz, 03.65.-w, 42.50.Lc

\section{INTRODUCTION}

Information about the state of a quantum system is usually obtained not from direct measurements on the system, but rather by monitoring its environment 1, 2, 3]. Therefore, the environment is not only a reservoir that selectively destroys quantum coherence, but also a "communication channel" through which observers find out about the system [4]. The formalism that ascribes to the system a time-dependent state deduced from a complete measurement of the environment - a quantum trajectory - has been introduced some time ago [5, 6, 7, 8]. Persistent monitoring of a quantum system by the environment can single out a preferred set of states, known as pointer states, which are the most robust in spite of the interaction with the environment, that is, least perturbed by it [9, 10]. In [1] we showed that under reasonable assumptions pointer states remain the most robust even when a single observer is performing continuous quantum measurement on a part of the environment to extract information about the system. Here we use the formalism of quantum trajectories [5, 6, 7, 8] to derive a set of conditional master equations which describe density matrices inferred by several observers simultaneously performing measurements on different parts of the environment. The knowledge a given observer has about the system, inferred from his measurement records, leads to his single observer density matrix. One can also consider a super-observer with access to all the records of all observers. His knowledge about the system is encapsulated in a super-observer density matrix. Both density matrices evolve according to conditional stochastic master equations that will be derived in the next Section.

The paper is organized as follows. In Section II we derive conditional stochastic master equations for the situation where multiple observers monitoring the environment of a quantum system try to infer its state from their measurement data. Section III discusses correlations between different measurement channels. In Section IV we apply the formalism to the quantum Brownian motion at zero temperature, a model for which coherent states are perfect pointer states. Observers trying to infer the state of the oscillator, initially prepared in a Schrdinger cat state $(|z\rangle+|-z\rangle) / \sqrt{2}$ of large amplitude coherent states, eventually fully agree when they measure in a basis correlated to the pointer states. In Sections V and VI we consider the model of resonance fluorescence of a two-level atom, for which pointer states do not really exist, and even the most predictable states are quite unpredictable. We discuss correlations between single observer state assignments for different measurement schemes, such as photo-detection and homodyne detection. Finally, Section VII contains our conclusions.

\section{CONDITIONAL MASTER EQUATIONS FOR SEVERAL OBSERVERS}

Imagine a system $\mathcal{S}$ coupled to an environment $\mathcal{E}$. The state of the environment is monitored by a set of detectors $\left\{\mathcal{D}_{i}\right\}(i=1, \ldots, C)$ in measurement basis that can be different for different detectors. When the results of these measurements are ignored (which in technical terms corresponds to tracing over $\mathcal{E}$ as well as over all the records of $\left\{\mathcal{D}_{i}\right\}$ ), the reduced density matrix of the system $\varrho(t)$ evolves according to an "unconditional" master equation (UME) 


$$
d \varrho(t)=d t \mathcal{L} \odot \varrho(t),
$$

within an infinitesimal time step $d t$. Here $\mathcal{L}$ is a linear super-operator acting on $\varrho$. This master equation is "unconditional" in the sense that all the information about the records of $\left\{\mathcal{D}_{i}\right\}$ has been ignored. Note that we are assuming that this evolution is Markovian, so that the state of the system at time $t+d t$ only depends on its state at time $t$. In what follows we shall make a still stronger assumption that the state of the fragments of the environment prior to the interaction with the system is always the same. In particular, the fragments of the environment that have interacted with $\mathcal{S}$ do not interact with it again. This quantum Markov approximation is accurate in a typical scattering situation. Then there is a natural distinction between input and output fields. The input field is assumed to be always described by the same density matrix $\mu$. The output field evolves in a way it must to account for its outward propagation. It is entangled with the system, and is eventually measured sufficiently far from the system so that the detection does not disturb the system. A spontaneous emission from, say, a two-level atom in free space is a particular example of the scattering situation where the input density matrix $\mu$ is a vacuum state.

\section{A. Multiple measurement channels}

Suppose that the measurement records are not ignored but, instead, as is typically the case, they are used to extract information about $\mathcal{S}$. Let us further assume that $\mathcal{E}$ includes parts $\left\{\mathcal{E}_{i}\right\}$ numbered by the index $i(i=1, \ldots, C)$ and that such parts are coupled to the detectors $\left\{\mathcal{D}_{i}\right\}$. These detectors correspond to different measurement channels. We model the measurements by detector $\mathcal{D}_{i}$ as a projection of the detector's state in a measurement basis with outcome $d N_{i}(t)$. In the example of spontaneous emission the parts $\left\{\mathcal{E}_{i}\right\}$ can be chosen as different directions of photon emission monitored by different detectors $\left\{\mathcal{D}_{i}\right\}$, while $d N_{i}(t)$ may be the number of photons collected by the detector $i$ during the infinitesimal time interval from $t$ to $t+d t$. No matter how much the measurement is delayed or how long it takes to decohere the state of the detector $\mathcal{D}_{i}$, the eventual outcome $d N_{i}(t)$ affects the knowledge about the state of the system at the time $t$ when the environment part $\mathcal{E}_{i}$ got entangled with $\mathcal{S}$. In general the measurement basis can be non-orthogonal and/or over-complete, but in the following, for the sake of simplicity, we restrict ourselves to the complete orthogonal case.

We assume that at $t=0$ the state of $\mathcal{S}$ was a density matrix $\varrho(0)$. As time goes on, at every time step $d t$ the system $\mathcal{S}$ is getting entangled with a new fragment of the environment $\mathcal{E}$. After interaction with $\mathcal{S}$ some of these fragments are not detected, while some other fragments, $\left\{\mathcal{E}_{i}\right\}$, are measured by the detectors $\left\{\mathcal{D}_{i}\right\}$. After time $t$ the initially uncorrelated state of $\mathcal{S}+\mathcal{E}+\mathcal{D}$ evolves into an entangled state $\rho_{\mathcal{S E D}}(t)$ (by $\mathcal{D}$ we are denoting the set of detectors $\left.\left\{\mathcal{D}_{i}\right\}\right)$. Ignoring the state of $\mathcal{E D}$ we get the unconditional density matrix

$$
\varrho(t)=\operatorname{Tr}_{\mathcal{E D}} \rho_{\mathcal{S E D}}(t),
$$

which evolves according to the UME, Eq. (11). Let us call $\mathcal{N}_{\alpha}(t)=\left(N_{1}(t), \ldots, N_{C}(t)\right)$ the set of strings of multiple channels measurement records. That is, $\mathcal{N}_{\alpha}(t)$ is a particular history of measurement results $\left\{d N_{i}\left(t^{\prime}\right)\right\}$ on all channels till time $t$, and the subscript $\alpha$ denotes a particular set of records. Then, the unconditional density matrix $\varrho(t)$ is a sum over all possible sets $\mathcal{N}_{\alpha}(t)$, namely

$$
\varrho(t)=\sum_{\mathcal{N}_{\alpha}(t)} \operatorname{Tr}_{\mathcal{E D}} \mathcal{P}_{\mathcal{N}_{\alpha}(t)} \rho_{\mathcal{S E D}}(t)
$$

Here $\mathcal{P}_{\mathcal{N}_{\alpha}(t)}=\mathcal{P}_{N_{1}(t)} \otimes \ldots \otimes \mathcal{P}_{N_{C}(t)}$ projects the state of all detectors according to the measurement records $N_{i}(t)(i=$ $1, \ldots, C)$. Different branches of the wave-function of $\mathcal{S E D}$ are labeled by different sets $\mathcal{N}_{\alpha}(t)$ of possible measurement records. A branch $\mathcal{N}_{\alpha}(t)$ has a probability

$$
p\left[\mathcal{N}_{\alpha}(t)\right]=\operatorname{Tr}_{\mathcal{S E D}} \mathcal{P}_{\mathcal{N}_{\alpha}(t)} \rho_{\mathcal{S E D}}(t) .
$$

A hypothetical "super-observer", who knows all the measurement records of all detectors, $\mathcal{N}_{\alpha}(t)=$ $\left(N_{1}(t), \ldots, N_{C}(t)\right)$, ascribes to $\mathcal{S}$ a state given by a multiple observers (or super-observer) conditional density matrix

$$
\rho\left[\mathcal{N}_{\alpha}(t)\right]=\frac{\operatorname{Tr}_{\mathcal{E D}} \mathcal{P}_{\mathcal{N}_{\alpha}(t)} \rho_{\mathcal{S E D}}(t)}{\operatorname{Tr}_{\mathcal{S}} \operatorname{Tr}_{\mathcal{E D}} \mathcal{P}_{\mathcal{N}_{\alpha}(t)} \rho_{\mathcal{S E D}}(t)}=\frac{\operatorname{Tr}_{\mathcal{E D}} \mathcal{P}_{\mathcal{N}_{\alpha}(t)} \rho_{\mathcal{S E D}}(t)}{p\left[\mathcal{N}_{\alpha}(t)\right]}
$$


normalized so that $\operatorname{Tr}_{\mathcal{S}} \rho\left[\mathcal{N}_{\alpha}(t)\right]=1$. From the point of view of the super-observer the set of records $\mathcal{N}_{\alpha}(t)$ actually happens. His description is necessarily probabilistic: at $t=0$ he could only calculate probabilities of different outcomes (Eq. (4) ), but could not predict his actual set of outcomes $\mathcal{N}_{\alpha}(t)$.

We assign to each measurement channel $i$ an observer $i$ who knows only his own record $N_{i}(t)$. He ascribes to $\mathcal{S}$ a state given by a single observer conditional density matrix

$$
\rho\left[N_{i}(t)\right]=\frac{\operatorname{Tr}_{\mathcal{E D}} \mathcal{P}_{N_{i}(t)} \rho_{\mathcal{S E D}}(t)}{\operatorname{Tr}_{\mathcal{S}} \operatorname{Tr}_{\mathcal{E D}} \mathcal{P}_{N_{i}(t)} \rho_{\mathcal{S E D}}(t)}
$$

conditioned only on his own record $N_{i}(t)$. It is easy to check that the single observer density matrix is an average over the super-observer density matrices with all records that are not known to the observer $i$. Indeed, denoting $\mathcal{N}_{\beta}^{N_{i}(t)}=\left(N_{1}(t), \ldots, N_{i}(t), \ldots, N_{C}(t)\right)$ a set of strings of multiple channel records that contain the particular record $N_{i}(t)$ in channel $i$, we have

$$
\rho\left[N_{i}(t)\right]=\sum_{\mathcal{N}_{\beta}^{N_{i}(t)}} \frac{p\left[\mathcal{N}_{\beta}^{N_{i}(t)}\right] \rho\left[\mathcal{N}_{\beta}^{N_{i}(t)}\right]}{p\left[N_{i}(t)\right]} .
$$

The probability distribution for the measurement record $N_{i}(t)$ is given by

$$
p\left[N_{i}(t)\right]=\operatorname{Tr}_{\mathcal{S E D}} \mathcal{P}_{N_{i}(t)} \rho_{\mathcal{S E D}}(t)=\sum_{\mathcal{N}_{\beta}^{N_{i}(t)}} p\left[\mathcal{N}_{\beta}^{N_{i}(t)}\right],
$$

which is analogous to Eq. (4).

The issue of compatibility of density matrices ascribed to a system by different observers was first considered by Peierls [12]. He noted that the state assignments of various observers cannot be arbitrarily different, and proposed that, in order to avoid contradiction between different state assignments, the product of the corresponding density matrices should be non-zero. A second condition put forward in [12], namely that the different density matrices should commute, was later shown to be too restrictive [13]. Necessary and sufficient condition for compatibility of several density matrices turns out to be simple [14]: Their supports must share at least one state (the support of a density matrix is the subspace spanned by all its eigenvectors with non zero eigenvalues).

In our setting the issue of compatibility of various single observer density matrices $\rho\left[N_{i}(t)\right]$ is settled very naturally. Indeed, the probability $p\left[\mathcal{N}_{\alpha}(t)\right]$ defined in Eq. (4) provides a measure of compatibility of different sets of outcomes: $p\left[\mathcal{N}_{\alpha}(t)\right]=0$ when records from different channels in the set $\mathcal{N}_{\alpha}(t)$ are mutually contradictory. It is easy to see that this condition is equivalent to the pre-requisite compatibility, i.e. the overlap of support [12, 13, 14]. Moreover, $p\left[\mathcal{N}_{\alpha}(t)\right]$ quantifies this compatibility, at least in the multiple observer setting we are about to investigate in more detail.

\section{B. Conditional master equations}

The evolution of the unconditional density matrix $\varrho(t)$ is determined by the unconditional master equation, Eq. (11). We now derive master equations that describe the evolution of the super-observer density matrix $\rho\left[\mathcal{N}_{\alpha}(t)\right]$, and of the density matrix $\rho\left[N_{i}(t)\right]$ of observer $i$, conditioned on their respective measurement results. Within the Markovian approximation, the master equation for the single observer conditional density matrix has the form [ 5 , 7]

$$
d \rho\left[\mathcal{N}_{\alpha}(t)\right]=d t \mathcal{L} \odot \rho\left[\mathcal{N}_{\alpha}(t)\right]+\mathcal{M}_{d \mathcal{N}_{\alpha}(t)} \odot \rho\left[\mathcal{N}_{\alpha}(t)\right] .
$$

In our case of the super-observer that "single observer" has access to all measurement records $\mathcal{N}_{\alpha}(t)$. The superoperator $\mathcal{M}_{d \mathcal{N}_{\alpha}(t)}$ conditions $\rho\left[\mathcal{N}_{\alpha}(t)\right]$ on present measurement results $d \mathcal{N}_{\alpha}(t)=\left(d N_{1}(t), \ldots, d N_{C}(t)\right)$ that are obtained at time $t$. It can be written as a sum of super-operators that depend on the measurement results on individual channels, $\mathcal{M}_{d \mathcal{N}_{\alpha}(t)}=\mathcal{M}_{d N_{1}(t)}+\ldots+\mathcal{M}_{d N_{C}(t)}$. Each super-operator $\mathcal{M}_{d N_{i}(t)}$ takes density operators to density operators and depends on the particular measurement strategy implemented in the measurement channel $i$. Examples of measurement strategies are point processes, such as photo-counting of optical fields, and diffusive processes, such as homodyne or heterodyne detection of optical fields. Also, $\mathcal{M}_{d \mathcal{N}_{\alpha}(t)} \odot \rho\left[\mathcal{N}_{\alpha}(t)\right]$ is nonlinear in $\rho\left[\mathcal{N}_{\alpha}(t)\right]$ and linear in the 
set of measurement results $d \mathcal{N}_{\alpha}(t)$. The nonlinearity comes into play in Eq. (5) when we normalize the density matrix. The action of this super-operator on $\rho\left[\mathcal{N}_{\alpha}(t)\right]$ is a generalization of the apparent "collapse of the wave-function" experienced by the super-observer confined to the branch $\mathcal{N}_{\alpha}(t)$. For a derivation of the super-operators $\mathcal{M}_{d N_{i}(t)}$ in terms of the projectors $\mathcal{P}_{N_{i}(t)}$ see the formalism of operations and effects described in [15, 16].

When measurement results $d \mathcal{N}_{\alpha}(t)$ are ignored, $\varrho(t)$ should follow the UME (Eq. (1)). In other words, the sum of the super-operator $\mathcal{M}_{d \mathcal{N}_{\alpha}(t)}$ over all possible strings of measurement results $d \mathcal{N}_{\alpha}(t)$ should vanish, that is:

$$
\sum_{d \mathcal{N}_{\alpha}(t)} \mathcal{M}_{d \mathcal{N}_{\alpha}(t)} \odot \rho\left[\mathcal{N}_{\alpha}(t)\right]=0
$$

We can also write down the master equation for the density matrix $\rho\left[N_{i}(t)\right]$ that observer $i$, who knows only his own records $N_{i}(t)$, ascribes to the system $\mathcal{S}$. Using Eqs.(79) we obtain

$$
d \rho\left[N_{i}(t)\right]=d t \mathcal{L} \odot \rho\left[N_{i}(t)\right]+\mathcal{M}_{d N_{i}(t)} \odot \rho\left[N_{i}(t)\right] .
$$

Indeed, this equation has the same form as that of the super-observer Eq. (9). The super-operator $\mathcal{M}_{d N_{i}(t)}$ depends only on the measurement result $d N_{i}(t)$ of observer $i$, and it is defined as an average over all records of all other observers unknown to him,

$$
\mathcal{M}_{d N_{i}(t)} \odot \rho\left[N_{i}(t)\right]=\sum_{\mathcal{N}_{\beta}^{N_{i}(t)}} \frac{p\left[\mathcal{N}_{\beta}^{N_{i}(t)}\right]}{p\left[N_{i}(t)\right]} \mathcal{M}_{d \mathcal{N}_{\beta}^{d N_{i}(t)} \odot \rho\left[\mathcal{N}_{\beta}^{N_{i}(t)}\right]}
$$

where $d \mathcal{N}_{\beta}^{d N_{i}(t)}$ is any string of multiple channel measurement record that contains the particular measurement results $d N_{i}(t)$ on channel $i$.

\section{CORRELATIONS BETWEEN DIFFERENT MEASUREMENT CHANNELS}

We study correlations between measurement records on different measurement channels, say channels $i$ and $j$. It is clear that in order to do so it is necessary to compare corresponding records $N_{i}(t)$ and $N_{j}(t)$. Therefore, we imagine there is someone who has access to the records on both channels, or the two observers with access to channels $i$ and $j$ communicate with each other and share their measurement records. Whatever the case is, we can think that there is a (super)observer who has access to the two measurement channels and whose string of records is $\left(N_{i}(t), N_{j}(t)\right)$. To follow the line of thought of previous sections, we will instead consider the super-observer who has access to all measurement channels, and whose string of records contain the particular record $N_{i}(t)$ on channel $i$, and the particular record $N_{j}(t)$ on channel $j$, i.e., his string of records is $\mathcal{N}_{\gamma}^{N_{i}(t), N_{j}(t)}=\left(N_{1}(t), \ldots, N_{i}(t), \ldots, N_{j}(t), \ldots, N_{C}(t)\right)$. Here the subscript $\gamma$ denotes a particular set of multiple channel records that contains records $N_{i}(t)$ and $N_{j}(t)$ in channels $i$ and $j$, respectively.

We define the average relative purity between the states ascribed to $\mathcal{S}$ by two such observers $i$ and $j$ as

$$
\begin{aligned}
O_{i j}(t) & =\sum_{N_{i}(t), N_{j}(t)} \sum_{\mathcal{N}_{\gamma}^{N_{i}(t), N_{j}(t)}} p\left[\mathcal{N}_{\gamma}^{N_{i}(t), N_{j}(t)}\right] \operatorname{Tr}_{\mathcal{S}} \rho\left[N_{i}(t)\right] \rho\left[N_{j}(t)\right] \\
& =\sum_{N_{i}(t), N_{j}(t)} p\left[N_{i}(t), N_{j}(t)\right] \operatorname{Tr}_{\mathcal{S}} \rho\left[N_{i}(t)\right] \rho\left[N_{j}(t)\right]
\end{aligned}
$$

where $p\left[N_{i}(t), N_{j}(t)\right]$ is the joint probability distribution for records $N_{i}(t)$ and $N_{j}(t)$, given by

$$
p\left[N_{i}(t), N_{j}(t)\right]=\sum_{\mathcal{N}_{\gamma}^{N_{i}(t), N_{j}(t)}} p\left[\mathcal{N}_{\gamma}^{N_{i}(t), N_{j}(t)}\right] .
$$

We also introduce the average relative purity $O_{i}(t)$ between the states of $\mathcal{S}$ ascribed by the observer $i$ and the superobserver, whose respective measurement records are $N_{i}(t)$ and $\mathcal{N}_{\beta}^{N_{i}(t)}$, i.e., the particular record $N_{i}(t)$ on channel $i$ 
is contained in the super-observer string of multiple channel records, $\mathcal{N}_{\beta}^{N_{i}(t)}=\left(N_{1}(t), \ldots, N_{i}(t), \ldots, N_{C}(t)\right)$. Here $\beta$ denotes a particular set of super-observer records that contains the record $N_{i}(t)$ in channel $i$. We define

$$
O_{i}(t)=\sum_{N_{i}(t)} \sum_{\mathcal{N}_{\beta}^{N_{i}(t)}} p\left[\mathcal{N}_{\beta}^{N_{i}(t)}\right] \operatorname{Tr}_{\mathcal{S}} \rho\left[N_{i}(t)\right] \rho\left[\mathcal{N}_{\beta}^{N_{i}(t)}\right] .
$$

Using Eqs.(7) (8) it is easy to check that this average relative purity equals the average purity of the state ascribed to $\mathcal{S}$ by observer $i$, and that it is also equal to the autocorrelation $O_{i i}(t)$ introduced in Eq. (13), namely

$$
O_{i}(t)=O_{i i}(t)=\sum_{N_{i}(t)} p\left[N_{i}(t)\right] \operatorname{Tr}_{\mathcal{S}} \rho^{2}\left[N_{i}(t)\right]
$$

In other words, an observer $i$ has no more information about the super-observer's records than the information already contained in his own records.

A better measure of correlations between density matrices is fidelity [17], which is defined as $F\left(\rho_{i}, \rho_{j}\right)=$ $\left\{\operatorname{Tr}\left[\left(\sqrt{\rho_{i}} \rho_{j} \sqrt{\rho_{i}}\right)^{1 / 2}\right]\right\}^{2}$. This can be easily calculated in two dimensions: $F\left(\rho_{i}, \rho_{j}\right)=\operatorname{Tr}\left(\rho_{i} \rho_{j}\right)+2\left(\operatorname{det} \rho_{i} \operatorname{det} \rho_{j}\right)^{1 / 2}$. Unfortunately it is more difficult to compute for more general cases, and for this reason we will use in the following relative purity as a measure of correlations.

In order to make a quantative study of correlations between different measurement channels it will be convenient to work in the framework of stochastic differential master equations. We first simplify our notation: we will denote the density matrix ascribed to the system by the super-observer, who has measurement records $\mathcal{N}_{\beta}^{N_{i}(t)}$, as

$$
\rho\left[\mathcal{N}_{\beta}^{N_{i}(t)}\right] \equiv \rho(t),
$$

and the density matrix of observer $i$, whose measurement record is $N_{i}(t)$, as

$$
\rho\left[N_{i}(t)\right] \equiv \rho_{i}(t)
$$

Also, we will denote the measurement records $\mathcal{N}_{\beta}^{N_{i}(t)}$ of the super-observer, and his measurement results $d \mathcal{N}_{\beta}^{d N_{i}(t)}$, that respectively contain the measurement record $N_{i}(t)$ and the measurement result $d N_{i}(t)$ on channel $i$, as

$$
\begin{aligned}
\mathcal{N}_{\beta}^{d N_{i}(t)} & \equiv \mathcal{N}(t), \\
d \mathcal{N}_{\beta}^{d N_{i}(t)} & \equiv d \mathcal{N}(t) .
\end{aligned}
$$

We emphasize again that, in the study of correlations, we consider the situation when the single channel measurement record $N_{i}(t)$ is contained in the super-observer records $\mathcal{N}_{\beta}=\mathcal{N}_{\beta}^{N_{i}(t)}$. Similarly, when we study correlations between measurement records on two different channels $i$ and $j$, we consider the situation when the two single channel measurement records $N_{i}(t)$ and $N_{j}(t)$ are both contained in the super-observer records $\mathcal{N}_{\beta}=\mathcal{N}_{\beta}^{N_{i}(t), N_{j}(t)}$.

The conditional master equation for the super-observer, that conditions his $\rho(t)$ on current measurement results $d \mathcal{N}(t)$, can be upgraded to a stochastic master equation (SME). A SME is a conditional master equation plus a probability distribution $P(d \mathcal{N}(t))$ for the measurement results $d \mathcal{N}(t)$. This probability distribution can be obtained from Eq. (4). $P[d \mathcal{N}(t)]$ is a conditional probability to get current measurement results $d \mathcal{N}(t)$ provided that the measurement records of the super-observer until time $t$ are $\mathcal{N}(t)$, that is

$$
P[d \mathcal{N}(t)] \equiv p[d \mathcal{N}(t) \mid \mathcal{N}(t)]=\frac{p[d \mathcal{N}(t), \mathcal{N}(t)]}{p[\mathcal{N}(t)]}
$$

where we used Bayes rule. Here $p[d \mathcal{N}(t), \mathcal{N}(t)]$ is the joint probability of having measurement records $\mathcal{N}(t)$ until time $t$, and of having measurement results $d \mathcal{N}(t)$ at time $t$. In the Markovian approximation $P[d \mathcal{N}(t)]$ depends on the records $\mathcal{N}(t)$ through the conditional density matrix $\rho(t)$,

$$
P[d \mathcal{N}(t)]=P[d \mathcal{N}(t) \mid \rho(t)] .
$$


The dependence of this probability distribution on the super-observer $\rho(t)$ leads to correlations between different measurement channels we are going to explore using a set of SMEs describing the stochastic evolutions for $\rho(t)$ and $\rho_{i}(t)$. This set of stochastic master equations is given by

$$
\begin{aligned}
d \rho(t) & =d t \mathcal{L} \odot \rho(t)+\mathcal{M}_{d \mathcal{N}(t)} \odot \rho(t), \\
d \rho_{i}(t) & =d t \mathcal{L} \odot \rho_{i}(t)+\mathcal{M}_{d N_{i}(t)} \odot \rho_{i}(t), \\
P\left[d N_{1}(t), \ldots, d N_{C}(t) \mid \rho(t)\right] & =\frac{\operatorname{Tr}_{\mathcal{S E D}} \mathcal{P}_{\mathcal{N}_{\alpha}(t), d \mathcal{N}_{\alpha}(t)} \rho_{\mathcal{S E D}}(t+d t)}{\operatorname{Tr}_{\mathcal{S E D}} \mathcal{P}_{\mathcal{N}_{\alpha}(t)} \rho_{\mathcal{S E D}}(t)} .
\end{aligned}
$$

We refer to this set of equations as a multiple channels stochastic master equation (MCSME).

We will use the above formalism to address questions regarding correlations between measurements on different channels:

- What is the average correlation between the density matrix of a single observer $\rho_{i}$ and the super-observer's density matrix $\rho$ ? We shall quantify this correlation by the average relative purity $O_{i}(t)$ defined in Eq. (15). As a short-hand, we will write it as $O_{i}(t)=\overline{\operatorname{Tr} \rho_{i}(t) \rho(t)}$, where the overline means the weighted average defined in Eq. (15). This relative purity is a measure of how different, on average, is the knowledge of the observer $i$ from the knowledge he would have had if he had access to the records of all the other observers. He cannot know more about the state $\rho(t)$ that the super-observer ascribes to the system that he can infer from his own measurement record only. The extracted information can be measured by the average purity of the single observer state $O_{i i}(t)=\overline{\operatorname{Tr} \rho_{i}^{2}(t)}$ (Eq. (16)). Even if the super-observer density matrix $\rho(t)$ had higher average purity, the average relative purity $O_{i}(t)$ would be equal to the single observer purity, $O_{i}(t)=O_{i i}(t)$, as derived in Eq. (16). This equality will be illustrated with several examples in Sections V and VI. $O_{i i}(t)$ is maximal for measurements in a basis correlated with the pointer states [11].

- What is the average correlation between different single observer density matrices $\rho_{i}$ and $\rho_{j}$ ? We shall quantify this correlation by the average relative purity $O_{i j}(t)$ defined in Eq. (13), that as a short-hand we write as $O_{i j}(t)=\overline{\operatorname{Tr} \rho_{i}(t) \rho_{j}(t)}$. In other words, how much do different observers agree about the state of the system? In Section IV we will show that for an initial Schrödinger cat state made of large amplitude coherent states (coherent states are perfect pointer states in the model of zero temperature quantum Brownian motion), and for measurements in a basis of the environment correlated with them, observers will, after an initial transient, reach full agreement, $O_{i j}(t \rightarrow \infty)=1$. Typically, as seen in the examples of Sections V and VI, the agreement is not perfect but it gets better when the observers' measurement basis get closer to those environmental states correlated to the pointer basis of the system. For resonance fluorescence from a two-level atom subjected to direct photo-detection (see Section V) we find an anti-correlation, $O_{i j}<1 / 2$. Each observer learns something about the state of the system but their estimates of the state $\rho_{i}(t)$ are anti-correlated. The two-level atom is very far from being classical and, what is more, photo-detection is very far from being a measurement in a basis correlated with the most predictable states.

\section{QUANTUM BROWNIAN MOTION}

\section{A. Correlation of the outcomes for pointer state measurements}

In this section we consider the well-known model of quantum Brownian motion consisting of a harmonic oscillator (the system) interacting with a reservoir of harmonic oscillators (phonons) with a position-position coupling. We will restrict ourselves to the case of a zero temperature environment. This model represents a damped harmonic oscillator. The self-Hamiltonian for the system is $H=\omega a^{\dagger} a$, where $\omega$ is the frequency of the oscillator and $a, a^{\dagger}$ are bosonic annihilation/creation operators. Imagine that a set of observers perform homodyne detection measurements on the environment of phonons so that each of them gains information about the state of the system oscillator. Given the set of records of all those observers, the MCSME of the system oscillator is [27]

$$
\begin{aligned}
& d \rho=d t\left(a \rho a^{\dagger}-\frac{1}{2} a^{\dagger} a \rho-\frac{1}{2} \rho a^{\dagger} a\right)+\sum_{i}\left(d N_{i}-\overline{d N_{i}(\rho)}\right)\left(\frac{(a+\gamma) \rho\left(a^{\dagger}+\gamma^{\star}\right)}{\operatorname{Tr}\left[(a+\gamma) \rho\left(a^{\dagger}+\gamma^{\star}\right)\right]}-\rho\right), \\
& d \rho_{i}=d t\left(a \rho_{i} a^{\dagger}-\frac{1}{2} a^{\dagger} a \rho_{i}-\frac{1}{2} \rho_{i} a^{\dagger} a\right)+\left(d N_{i}-\overline{d N_{i}\left(\rho_{i}\right)}\right)\left(\frac{(a+\gamma) \rho_{i}\left(a^{\dagger}+\gamma^{\star}\right)}{\operatorname{Tr}\left[(a+\gamma) \rho_{i}\left(a^{\dagger}+\gamma^{\star}\right)\right]}-\rho_{i}\right),
\end{aligned}
$$




$$
\overline{d N_{i}(\rho)}=\eta_{i} d t\left[R^{2}+R e^{-i \phi} \operatorname{Tr} \rho a+R e^{+i \phi} \operatorname{Tr} \rho a^{\dagger}+\operatorname{Tr} \rho a^{\dagger} a\right] .
$$

These equations for the conditional evolution of the density matrices of the system, written in the interaction picture representation, are valid in the rotating wave approximation. Here we use Itô version of stochastic calculus. The first terms on the RHS of Eqs. (25126) are of Lindblad form and describe damping and decoherence due to spontaneous emission of phonons. We have set the damping coefficient to one. The second (stochastic) terms feed back into the master equation information about the state of the system gained by observers. The coefficient $\gamma=R \exp (i \phi)$ is the amplitude of the local oscillator in the homodyne detector [18]. For simplicity, we are asumming here that all observers perform the same kind of homodyne detection, so that the amplitudes $R_{i}$ and phases $\phi_{i}$ are all equal. We will lift this restriction in later examples. The number of phonons detected by observer $i$ in an infinitesimal interval from $t$ to $t+d t$ is $d N_{i}(t) \in\{0,1\}$, with an average given by Eq. (27) and $\overline{d N_{i} d N_{j}}=\delta_{i j} \overline{d N_{i}}$. The efficiencies $\eta_{i}$ of different detectors can be defined as the fractions of phonons monitored by particular detectors. In the phonodetection limit $(R=0)$, the average detection rate Eq. (27) is proportional to the average occupation number. Whenever a phonon is detected $\left(d N_{i}=1\right.$ for any $\left.i\right)$ the occupation numbers in $\rho$ are reduced by one. In the homodyne limit $(R \gg 1)$ the detection rates measure the coherent amplitude $\operatorname{Tr} \rho\left(e^{+i \phi} a+e^{-i \phi} a^{\dagger}\right)$ of the state of the system.

To illustrate how different observers are gaining information about the system and how correlations between different measurement channels arise in the process of continuous measurement we consider superpositions of large amplitude coherent states. According to the exact solution [20] coherent states $\left|z_{0}\right\rangle$, such that $a\left|z_{0}\right\rangle=z_{0}\left|z_{0}\right\rangle$, decay to the ground state like $\left|z_{0} e^{-t}\right\rangle=|z\rangle$ without producing any entropy. At $T=0$ they are the perfect pointer states of the quantum Brownian motion model [19]. The decay to the ground state takes place on a time scale of the order of the damping rate, which we have set to 1 . In a subspace spanned by $|+z\rangle$ and $|-z\rangle$ a general density matrix is

$$
\rho(t)=\frac{1+A(t)}{2}|+z\rangle\left\langle+z\left|+\frac{1-A(t)}{2}\right|-z\right\rangle\langle-z|+C(t)|+z\rangle\left\langle-z\left|+C^{*}(t)\right|-z\right\rangle\langle+z| .
$$

Substitution of this density matrix into Eq. [25), and subsequent left and right projections on $| \pm z\rangle$ [28] give stochastic differential equations for $A(t)$ and $C(t)$. $C(t)$ decays to 0 on a decoherence timescale which in our subspace of $| \pm z\rangle=| \pm r \exp (i \psi)\rangle$ is given by $1 / r^{2}$. For initial $r \gg 1$ this decoherence is much faster than damping and it takes place much before the states $| \pm z\rangle$ decay to the ground state. In the opposite case of $r \ll 1$ the states $| \pm z\rangle$ decay to the ground state before they can be distinguished by the environment. Both limits were considered in Ref. [20]. Coherent states with coherent amplitudes $\pm z$ that differ less than 1 cannot be distinguished. Here we concentrate on the distiguishable case of $r \gg 1$. In this limit we can self-consistently ignore damping and focus on the decoherence and measurement process. In the homodyne limit $(R \gg r)$, where detection rates are fast as compared to the spontaneous emission (decoherence) time, at any given time the correlators for the increments $d n_{i} \equiv d N_{i}-\overline{d N_{i}(\rho)}$ are

$$
\overline{\overline{d n_{i}}=0}, \overline{d n_{i} d n_{j}} \approx \delta_{i j} \overline{d N_{i}} \approx \delta_{i j} \eta_{i} R^{2} d t+\mathcal{O}(R) .
$$

In this limit the increments can be approximated by $d n_{i}=\sqrt{\eta_{i}} R d W_{i}$, where $d W_{i}$ 's are gaussian Wiener increments, such that $\overline{d W_{i}}=0$ and $\overline{d W_{i} d W_{j}}=\delta_{i j} d t[6$, , 7]. After introducing a variable $B$ as $A=\tanh B$, and translating to Stratonovich convention, the super-observer's equation for $B$ reads

$$
\frac{d B}{d \tau}=\eta \tanh B+\sum_{i} \sqrt{\eta_{i}} \theta_{i}(\tau)
$$

where we have defined a new time variable $\tau=4 t r^{2} \cos ^{2}(\phi-\psi)$ and $\eta=\sum_{i} \eta_{i}$. Here $\theta_{i}$ are stochastic continuous functions of time, defined as $d W_{i}=\theta_{i} d t$. These stochastic functions are white noises with correlators

$$
\overline{\theta_{i}\left(\tau_{1}\right) \theta_{j}\left(\tau_{2}\right)}=\delta_{i j} \delta\left(\tau_{1}-\tau_{2}\right) .
$$

According to Eq. (30), $B$ initially performs a random walk driven by the noises but once it diffuses into a positive $(\tanh B=+1)$ or negative ( $\tanh B=-1$ ) domain, the deterministic force $\eta \tanh B$ takes over and inevitably drives $B$ towards positive or negative infinity. After the transient time $\tau \sim 1 / \eta, A$ settles down at $A= \pm 1$ which corresponds to the pure state $| \pm z\rangle$ (see the super-observer's trajectory $A(\tau)$ in Fig.1). By this time an observer who knows all 


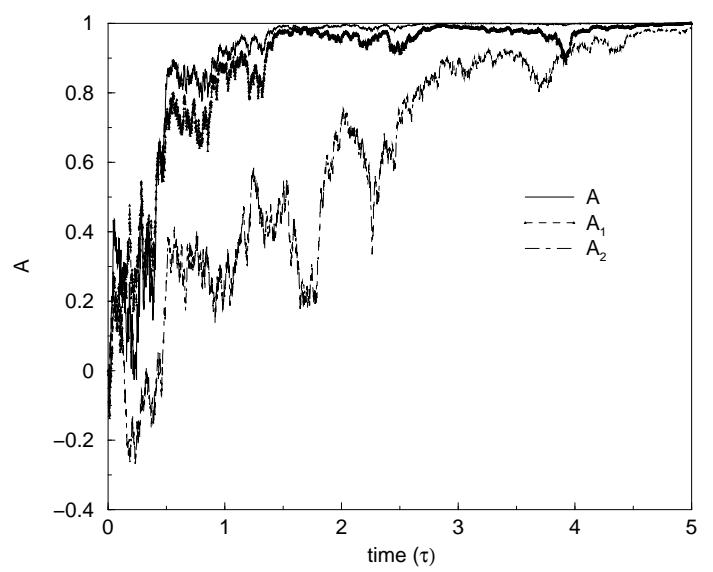

FIG. 1: A single realization of the stochastic trajectories $A(\tau)$ (thick line), $A_{1}(\tau)$ and $A_{2}(\tau)$ (thin lines) for $\eta_{1}=0.7$ and $\eta_{2}=0.3$. The super-observer's $A(\tau)$ settles at +1 around $\tau \approx 1$, it is followed by $A_{1}(\tau)$ after a $\tau$-delay $\approx 1$. $A_{2}(\tau)$ after a long period of indecision settles down at +1 at $\tau \approx 5$.

$d N_{i}(t)$ can tell whether the system oscillator is in the state $|z\rangle$ or $|-z\rangle$, and attributes to the system the appropriate pure state. This happens also when the total efficiency $\eta$ is less than one.

An observer $i$ ascribes to the system a state $\rho_{i}$ conditioned on his own records $d N_{i}(t)$ only. Since we want to study correlations between the measurement records, the evolution of $\rho_{i}$ is given by the MCSME (Eq. (26)). Taking the homodyne $R \gg r$ limit in the single observer case we get

$$
d N_{i}-\overline{d N_{i}\left(\rho_{i}\right)}=\left(d N_{i}-\overline{d N_{i}(\rho)}\right)+\left(\overline{d N_{i}(\rho)}-\overline{d N_{i}\left(\rho_{i}\right)}\right) \stackrel{R \gg r}{\approx} \sqrt{\eta_{i}} R d W_{i}+2 \eta_{i} r R\left(A-A_{i}\right) \cos (\psi-\phi) .
$$

Just as for the case of the super-observer (Eq. (25)), substitution of the ansatz Eq. (28) into Eq. (26) and neglecting any $\mathcal{O}(1 / R)$ terms, leads to the equation for the single observer $B_{i}$,

$$
\frac{d B_{i}}{d \tau}=\eta_{i} \tanh B_{i}+\left[\eta_{i} \tanh B-\eta_{i} \tanh B_{i}+\sqrt{\eta_{i}} \theta_{i}\right]=\eta_{i} \tanh B+\sqrt{\eta_{i}} \theta_{i},
$$

where $A_{i}=\tanh B_{i}$. The terms in the square brackets come from the stochastic term in Eq. (26). Note that the super-observer's $B$ appears in the evolution equation of the $B_{i}$ associated to the single measurement channel $i$. This reflects the fact that the single channel and multiple channels measurement results are correlated in the MCSME (Eq. (24)).

Let us now study how the evolutions of $A$ according to the super-observer and single observers are correlated. On the one hand, according to Eq. (30), the super-observer evolution settles $A=\tanh B$ at \pm 1 after the transient time $\tau \sim 1 / \eta$. On the other hand, the single observer evolution is given by Eq. (33), and correlations between the two evolutions enter through the first term in the most right hand side of that equation containing the super-observer $A=\tanh B$. Once $A=\tanh B= \pm 1$ is chosen, the deterministic drift term $\eta_{i} \tanh B$ on the RHS of Eq. (33) will inevitably force $A_{i}=\tanh B_{i}$ to make the same choice after the longer transient time $\tau \sim 1 / \eta_{i}$. Eventually all observers will settle down at $A=A_{i}= \pm 1$, and the average relative purities will be equal to one, $O_{i}=O_{i j}=1$ (see the single realizations for two measurement channels $i=1,2$ in Fig.1).

In our example the observers finally find out which of the two coherent states is the state of the system. It is possible because the initial coherent states have large amplitudes $\pm z$ with $|z| \gg 1$ so that the decoherence time is much shorter than the spontaneous emission time. In the opposite regime of $|z| \ll 1$ the decoherence time is longer than the spontaneous emission time, and the observers will not find out the state before it decays to the vacuum state.

\section{B. Independence of the outcome distributions}

To have a better feeling of the multiple channels stochastic master equation (MCSME) formalism we consider the following example. There are two observers $i=1,2$. The measurements by observer 2 affect the evolution of the super-observer's density matrix $\rho$. Since the environment is also monitored by observer 1 , in principle observer 1 
may be able to identify perturbations of $\rho$ produced by measurements of observer 2 and realize that there is another observer monitoring the system. In Section II we gave a general argument that, as a direct consequence of the quantum Markov approximation, observer 2 cannot find out if there is another observer. Here we present a simple calculation which illustrates this fact in our example of superposition of coherent states.

To begin with, note that Eq. (30) is equivalent to the following Fokker-Planck equation for the probability distribution $P(\tau, B)$ for $B$ at time $\tau$ [23],

$$
\frac{1}{\eta} \frac{\partial P}{\partial \tau}=-\frac{\partial}{\partial B} \tanh (B) P+\frac{1}{2} \frac{\partial^{2}}{\partial B^{2}} P .
$$

We can compare the following two situations:

1) Observer 1 is the only observer or $\eta_{2}=0$. His probability distribution evolves according to Eq. (34). The initial condition $P\left(0, B_{1}\right)=\delta\left(B_{1}\right)$ leads to the solution

$$
P^{(1)}\left(\tau, B_{1}\right)=\frac{\exp \left(-\frac{\left(B_{1}-\eta_{1} \tau\right)^{2}}{2 \eta_{1} \tau}\right)}{\sqrt{2 \pi \eta_{1} \tau}}+\frac{\exp \left(-\frac{\left(B_{1}+\eta_{1} \tau\right)^{2}}{2 \eta_{1} \tau}\right)}{\sqrt{2 \pi \eta_{1} \tau}} .
$$

2) There is an observer 2 with $\eta_{2} \gg \eta_{1}$. In this limit, where the perturbations by observer 2 are the strongest, one is most likely to suspect that the less efficient observer 1 could find out about the more efficient observer 2 . The evolution of $B(\tau)$ is mainly conditioned upon the measurements of observer 2 . The multiple observer $A$ settles at \pm 1 on a time-scale $1 / \eta$ which is much faster than the time $1 / \eta_{1}$ observer 1 needs to find out about the system. The state of the system is settled without any influence of measurements of observer 1 . Suppose that, with the probability $1 / 2$, the multiple observer state $\tanh B=+1$ was chosen. $B_{1}$ evolves according to Eq. (33) with a fixed $\tanh B=+1$. The probability distribution for $B_{1}$ is

$$
P_{+}\left(\tau, B_{1}\right)=\frac{\exp \left(-\frac{\left(B_{1}-\eta_{1} \tau\right)^{2}}{2 \eta_{1} \tau}\right)}{\sqrt{2 \pi \eta_{1} \tau}} .
$$

Also with the probability $1 / 2, \tanh B=-1$ can be chosen. Now $\tanh B=-1$ is fixed in Eq. (33) and the probability distribution is

$$
P_{-}\left(\tau, B_{1}\right)=\frac{\exp \left(-\frac{\left(B_{1}+\eta_{1} \tau\right)^{2}}{2 \eta_{1} \tau}\right)}{\sqrt{2 \pi \eta_{1} \tau}} .
$$

As we do not know which super-observer's state will be chosen, the two probabilities add to give $P^{(2)}=P_{+}+P_{-}$. It is easy to check that $P^{(2)}=P^{(1)}$ in Eq. (35). The probability distributions coincide, so observer 1 cannot find out if there is any observer 2 even if observer 1 detects just $1 \%$ of phonons and the other more efficient observer detects $99 \%$ or almost all phonons.

\section{TWO-LEVEL ATOM: DIRECT PHOTO-DETECTION}

We want to contrast the quantum Brownian motion model with an example of a system with a small Hilbert space, such as a driven two-level atom coupled to the radiation field, for which we do not expect perfect pointer states. In Appendix A we derive the MCSME for a two-level atom driven by a laser beam with frequency $\omega$ and whose emitted radiation is subjected to photo-detection. It takes the form

$$
\begin{aligned}
& d \rho=-i d t\left[\omega \sigma_{x}, \rho\right]+d t\left(c \rho c^{\dagger}-\frac{1}{2} c^{\dagger} c \rho-\frac{1}{2} \rho c^{\dagger} c\right)+\sum_{i}\left(d N_{i}-\overline{d N_{i}(\rho)}\right)\left(\frac{c \rho c^{\dagger}}{\operatorname{Tr}\left[c \rho c^{\dagger}\right]}-\rho\right) \\
& d \rho_{i}=-i d t\left[\omega \sigma_{x}, \rho_{i}\right]+d t\left(c \rho_{i} c^{\dagger}-\frac{1}{2} c^{\dagger} c \rho_{i}-\frac{1}{2} \rho_{i} c^{\dagger} c\right)+\left(d N_{i}-\overline{d N_{i}\left(\rho_{i}\right)}\right)\left(\frac{c \rho_{i} c^{\dagger}}{\operatorname{Tr}\left[c \rho_{i} c^{\dagger}\right]}-\rho_{i}\right) \\
& \overline{d N_{i}(\rho)}=\eta_{i} d t \operatorname{Tr}\left[\rho c^{\dagger} c\right] .
\end{aligned}
$$


The density matrix $\rho$ of the atom is a $2 \times 2$ matrix

$$
\rho=\frac{1}{2}\left[I+x \sigma_{x}+y \sigma_{y}+z \sigma_{z}\right] .
$$

The lowering operator is $c=\left(\sigma_{x}-i \sigma_{y}\right) / 2$, and the number of photons detected in channel $i$ between $t$ and $t+d t$ is $d N_{i} \in\{0,1\}$ with an average proportional to the occupation number of the atom, see Eq. (40), and $\overline{d N_{i} d N_{j}}=\delta_{i j} \overline{d N_{i}}$. Following each detection of a photon (any $d N_{i}=1$ ), the atom is known to be in the ground state (the -1 eigenstate of $\sigma_{z}$ ), from where it is excited again by a laser beam through the Hamiltonian term $\omega \sigma_{x}$. The efficiency $\eta_{i}$ of the detector used by observer $i$ is the fraction of photons which are detected by him.

When $\omega \gg 1$ the most predictable states of the two-level atom are $\sigma_{x}$ eigenstates, i.e., they are determined by the Hamiltonian $\omega \sigma_{x}$ describing the excitations via the laser beam 24]. These states are far from perfect since they have a nonzero initial rate of purity loss. Moreover, while eigenstates of $\sigma_{x}$ are most predictable, they are not the most effective in making an imprint on the environment (as real pointer states should be [4, 9, 10]. In particular, the environment-system Hamiltonian does not preserve them. As a consequence, we do not expect agreement between observers even if they are measuring in a basis of the environment correlated to the $\sigma_{x}$-eigenstates of the atom. Direct photo-detection is a way to find out if the atom is in the ground state. This state is complementary to the most predictable states. That is why we expect the relative purity between observers to be very poor. In fact we will find any two observers to be anti-correlated, $O_{i j}<1 / 2$.

\section{A. The $\omega \gg 1$ limit}

For $\omega \gg 1$ Eqs. (38 39) can be solved rigorously. Suppose that no photons are detected for a certain period of time, $d N_{i}(t)=0$. During this time the density matrix $\rho$ in Eq. (41) evolves according to the deterministic part of Eq. (38). The unitary self-evolution with the Hamiltonian $\omega \sigma_{x}$ is mixing $y$ and $z$ with the frequency $2 \omega$. It is convenient to use the interaction picture, where

$$
\begin{aligned}
& x=x_{\text {int }}, \\
& y=y_{\text {int }} \cos 2 \omega t-z_{\text {int }} \sin 2 \omega t, \\
& z=y_{\text {int }} \sin 2 \omega t+z_{\text {int }} \cos 2 \omega t,
\end{aligned}
$$

and the variation in time of $x_{\mathrm{int}}, y_{\mathrm{int}}, z_{\mathrm{int}}$ is slow as compared to $\omega$. When we substitute the density matrix Eq. (41) into the deterministic part of Eq. (38), use the interaction picture, and average over one period of oscillation with frequency $\omega$, we will obtain the following equations [29]

$$
\begin{aligned}
\frac{d x_{\mathrm{int}}}{d t} & =-\frac{1}{2} x_{\mathrm{int}}, \\
\frac{d y_{\mathrm{int}}}{d t} & =-\frac{3}{4}(1-\eta) y_{\mathrm{int}}, \\
\frac{d z_{\mathrm{int}}}{d t} & =-\frac{3}{4}(1-\eta) z_{\mathrm{int}} .
\end{aligned}
$$

Every time a photon is detected the super-observer density matrix $\rho$ is projected to the ground state. All the information about the previous evolution of $\rho(t)$ is forgotten. Suppose that a detection took place at time $t=0$. Just after the detection the initial conditions are $x\left(0^{+}\right)=0, y\left(0^{+}\right)=0$ and $z\left(0^{+}\right)=-1$. Before the next detection happens, $x, y, z$ evolve according to Eqs.4243,

$$
\begin{aligned}
& X(t)=0 \\
& Y(t)=e^{-\frac{3}{4}(1-\eta) t} \sin 2 \omega t \\
& Z(t)=-e^{-\frac{3}{4}(1-\eta) t} \cos 2 \omega t
\end{aligned}
$$

where $t$ is the time elapsed since the last photo-detection. This solution is valid until the next detection takes place. The next detection at $t=t_{d}$ will bring $\rho$ to the ground state again, from where the system will be excited according 
to $x=X\left(t-t_{d}\right), y=Y\left(t-t_{d}\right), z=Z\left(t-t_{d}\right)$. The probability that an observer $i$ will detect a photon between $t$ and $t+d t$ after the last detection by any observer is

$$
\overline{d N_{i}(\rho(t))}=d t \eta_{i} \frac{1+Z(t)}{2}
$$

The above argument can also be applied to Eq. (39). Every time an observer $i$ detects a photon his state $\rho_{i}$ jumps to the ground state, from where it is excited according to

$$
\begin{aligned}
X_{i}(t) & =0, \\
Y_{i}(t) & =e^{-\frac{3}{4}\left(1-\eta_{i}\right) t} \sin 2 \omega t, \\
Z_{i}(t) & =-e^{-\frac{3}{4}\left(1-\eta_{i}\right) t} \cos 2 \omega t .
\end{aligned}
$$

The time $t$ here is the time since the last detection by the observer $i$.

\section{B. Distribution of waiting times}

In this example we shall see again that a single observer cannot find out if there is any other observer. We will consider just two observers $i=1,2$ and we will derive the distribution of waiting times (times between subsequent detections) for observer 1 . We will show that this distribution does not depend on $\eta_{2}$ so it is not sensitive to the presence or absence of any observer 2. Any higher order correlations between detection times can be expressed by this distribution of waiting times because every time a photon is detected by observer 1 , the atomic state goes down to the ground state so that any history before the detection does not affect evolution that follows that detection. The distribution of waiting times contains all the information observer 1 can possibly extract from his measurements.

Suppose that observer 1 detects a photon at time $t=0$. What is the probability $w_{1}(\tau)$ that he will detect the next photon at time $t=\tau$ ? If observer 1 were the only observer, so that $\eta_{1}=\eta$, then the answer would be

$$
w_{1}(\tau)=\left(\eta_{1} \frac{1+Z_{1}(\tau)}{2}\right) e^{-\int_{0}^{\tau} d \tau_{1} \eta_{1} \frac{1+Z_{1}\left(\tau_{1}\right)}{2} \omega \gg 1} \frac{\eta_{1}}{2} e^{-\frac{\eta_{1} \tau}{2}} .
$$

The first factor is the average detection rate Eq. (45), and the second one is the probability that no photon is detected between 0 and $\tau$. As it should be, $w_{1}(\tau)$ is normalized to unity. To obtain the final expression for $w_{1}(\tau)$ in Eq. (47) we have neglected all terms which vanish for $\omega \gg 1$ as well as fast oscillating terms $\sim \cos 2 \omega \tau$.

If there is a second observer, then the detection rate of observer $i$ depends not on $z_{\alpha}(t)$ but on $z(t)$ (see Eq. (45)). In general there may be $n=0,1, \ldots, \infty$ detections by observer 2 between 0 and $\tau$. Every time there is a detection by observer 2 at $t=t_{j},(j=1, \ldots, n), z(t)$ jumps down to -1 . For $t_{j}<t<t_{j+1}$ it evolves as $z(t)=Z\left(t-t_{j}\right)$. The probability that there is no detection by observer 1 between times 0 and $\tau$, given that there are $n$ detections by observer 2 at the times $t_{1}, \ldots, t_{n}$, is given by

$$
D_{n}\left(t_{1}, \ldots, t_{n}, \tau\right)=q_{2}\left(t_{1}\right) q_{2}\left(t_{2}-t_{1}\right) \ldots q_{2}\left(t_{n}-t_{n-1}\right) e^{-\frac{\eta}{2}\left(\tau-t_{n}\right)}
$$

where $q_{2}(\tau)$ is distribution of waiting times for observer 2 given that there are no detections by 1 ,

$$
q_{2}(\tau) \stackrel{\omega \gg 1}{\approx} e^{-\frac{\eta}{2} \tau} \frac{\eta_{2}}{2}\left(1-e^{-\frac{3}{4}(1-\eta) \tau} \cos 2 \omega \tau\right)
$$

and the factor $e^{-\frac{\eta}{2}\left(t-t_{n}\right)}$ is the probability that no detections by any observer take place between $t_{n}$ and $t$. The distribution of waiting times for observer 1, averaged over detections by observer 2, is given by $D_{n}$ multiplied by the detection rate of observer 1 at $\tau$, and averaged over all possible $n$ and $t_{1}, \ldots, t_{n}$. Therefore the final expression for the waiting time distribution $f_{\text {wait }}(\tau)$ for observer 1 in the presence of detections by observer 2 is given by

$$
f_{\text {wait }}(\tau)=\sum_{n=0}^{\infty} \int_{0}^{\tau} d t_{1} \int_{t_{1}}^{\tau} d t_{2} \ldots \int_{t_{n-1}}^{\tau} d t_{n} D_{n}\left(t_{1}, \ldots, t_{n}, \tau\right) \frac{\eta_{1}}{2}\left[1+Z\left(\tau-t_{n}\right)\right] \stackrel{\omega \gg 1}{\approx} \frac{\eta_{1}}{2} e^{-\frac{\eta_{1}}{2} \tau}
$$


where, again, we have neglected terms which vanish for $\omega \gg 1$ and any fast oscillating terms. In Appendix B we show how to obtain this last formula. We conclude that the distribution of waiting times for observer 1 in the presence of detections by observer 2 (Eq. (50) is the same as that for no observer 2 present (Eq. (47)). The distribution of waiting times for observer 1 is not sensitive to observer 2 .

\section{Average relative purity between $\rho_{1}$ and $\rho$}

Let us now find out how much does a given observer, say $i=1$, know about the state of the super-observer. To this end we will calculate the average relative purity between the single observer and the super-observer density matrices, $O_{1}=\overline{\operatorname{Tr} \rho_{1} \rho}$. Imagine the following situation. Take an arbitrary instant of time $\tau=0$ and call $o_{1}^{(n)}(\tau=0)=\operatorname{Tr} \rho_{1}(\tau=$ 0) $\rho(\tau=0)$ the relative purity given that the last detection of observer 1 took place at $\tau=-t$ and there were $n$ detections by observer 2 between $\tau=-t$ and $\tau=0$. Then the average relative purity $O_{1}$ evaluated at the time $\tau=0$ will be equal to the $t$-average (i.e., average over all possible initial times of detection by 1 ) of the relative purity $o_{1}^{(n)}(\tau=0)$ given that there were no detections by observer 1 between $\tau=-t$ and $\tau=0$ and averaged over all the possible numbers $n$ of detections by observer 2 and his detection times $t_{1}, \ldots, t_{n}$. For the sake of clarity, we now shift the time origin as $\tau \rightarrow \tau+t$, so that the last detection of 1 took place at time 0 and we are interested in evaluating $O_{1}$ at time $t$. The unnormalized probability distribution for no detections by observer 1 between 0 and $t$, and $n$ detections by observer 2 at the times $t_{1}, \ldots, t_{n}$ is $D_{n}\left(t_{1}, \ldots, t_{n}, t\right)$, given by Eq. (48). The normalizing factor for this distribution is

$$
n_{1}=\sum_{n=0}^{\infty} \int_{0}^{\infty} d t \int_{0}^{t} d t_{1} \int_{t_{1}}^{t} d t_{2} \ldots \int_{t_{n-1}}^{t} d t_{n} D_{n}\left(t_{1}, \ldots, t_{n}, t\right) \stackrel{\omega \gg 1}{\approx} \frac{2}{\eta_{1}}
$$

Given that the last detection by observer 1 took place at time 0 and the last detection by any observer happened at time $t_{n}$, the relevant relative purity is

$$
o_{1}^{(n)}(t)=\operatorname{Tr} \rho_{1}(t) \rho(t)=\frac{1}{2}+\frac{1}{2} e^{-\frac{3}{4}\left(1-\eta_{1}\right) t} e^{-\frac{3}{4}(1-\eta)\left(t-t_{n}\right)} \cos 2 \omega t_{n} .
$$

This relative purity, when averaged with the probability distribution (48), gives

$$
O_{1}=n_{1}^{-1} \sum_{n=0}^{\infty} \int_{0}^{\infty} d t \int_{0}^{t} d t_{1} \int_{t_{1}}^{t} d t_{2} \ldots \int_{t_{n-1}}^{t} d t_{n} D_{n}\left(t_{1}, \ldots, t_{n}, t\right) o_{1}^{(n)}(t)=\frac{1}{2}+\frac{\eta_{1}}{2\left(3-2 \eta_{1}\right)}
$$

where we have neglected all $\mathcal{O}(1 / \omega)$ terms.

On the other hand, the average purity gained by observer 1 can be calculated as follows. According to Eqs. (46), the purity at the time $t$ after the last detection is $o_{11}(t)=\operatorname{Tr} \rho_{1}^{2}(t)=\frac{1}{2}+\frac{1}{2} \exp \left[-\frac{3}{2}\left(1-\eta_{1}\right) t\right]$. The probability that there was no detection between 0 and $t$ is $\exp \left(-\eta_{1} t / 2\right)$. Therefore, the average purity $O_{11}$ is $o_{11}(t)$ averaged over $t$ :

$$
O_{11}=\frac{\int_{0}^{\infty} d t e^{-\frac{\eta_{1}}{2} t} o_{11}(t)}{\int_{0}^{\infty} d t e^{-\frac{\eta_{1}}{2} t}}=\frac{1}{2}+\frac{\eta_{1}}{2\left(3-2 \eta_{1}\right)} .
$$

As expected from Eq. (16), $O_{11}$ coincides with $O_{1}$ (see Fig.2). Let us now comment on the limiting cases $\eta_{1}=0$ and $\eta_{1}=1$. In the former case we get $O_{11}=0.5$, that corresponds to no information gain by the observer ( $\rho_{1}$ is maximally mixed). In the latter case we get $O_{11}=1$, that is maximal gain of information, and $\rho_{1}$ is pure.

\section{Average relative purity $O_{i j}$}

The average relative purity $O_{12}=\overline{\operatorname{Tr} \rho_{1} \rho_{2}}$ has contributions from the following two situations:

(1) The last detection by observer 1 took place at time 0 . Between times 0 and $t$ there were $n \geq 1$ detections by observer 2 at the times $t_{1}, \ldots, t_{n}$. The last detection before $t$ was made by observer 2 at time $t_{n}$. According to Eqs.(44), the relative purity at $t$ is 


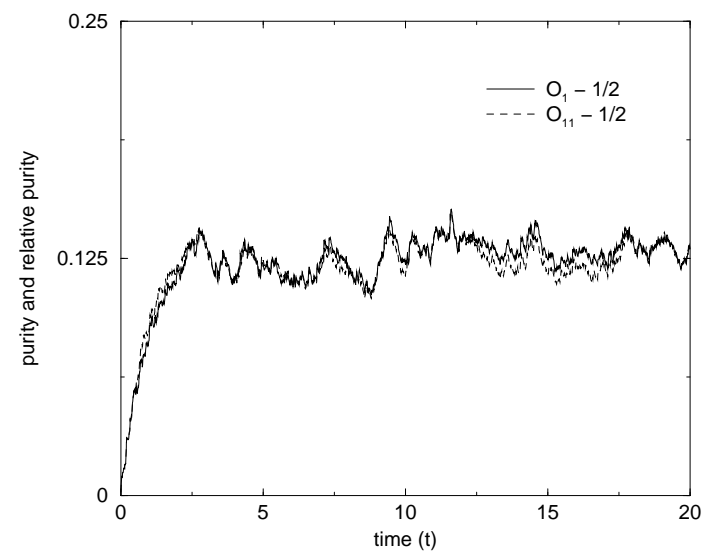

FIG. 2: Average purity $O_{11}-1 / 2$ and average relative purity $O_{1}-1 / 2$ for an observer performing photo-detection measurements. The initial condition is maximal lack of knowledge, i.e. $\rho(t=0)=\rho_{1}(t=0)=I / 2$. The super-observer's efficiency is $\eta=0.6$ and the single observer one is $\eta_{1}=0.5$. According to Eqs. (53] [54 the asymptotic value is $O_{1}(\infty)=O_{11}(\infty)=0.125$. The stochastic trajectories are an average over 256 single realizations. The small discrepancy between $O_{1}$ and $O_{11}$ in the figure is an artifact of the finite number of realizations used for calculating the averages.

$$
o_{12}^{(n)}(t)=\operatorname{Tr} \rho_{1}(t) \rho_{2}(t)=\frac{1}{2}+\frac{1}{2} e^{-\frac{3}{4}\left(1-\eta_{1}\right) t} e^{-\frac{3}{4}\left(1-\eta_{2}\right)\left(t-t_{n}\right)} \cos 2 \omega t_{n} .
$$

The normalizing factor for the probability distribution is

$$
n_{12}=\sum_{n=1}^{\infty} \int_{0}^{\infty} d t \int_{0}^{t} d t_{1} \int_{t_{1}}^{t} d t_{2} \ldots \int_{t_{n-1}}^{t} d t_{n} D_{n}\left(t_{1}, \ldots, t_{n}, t\right) \stackrel{\omega \gg 1}{\approx} \frac{2 \eta_{2}}{\eta_{1}\left(\eta_{1}+\eta_{2}\right)}
$$

and the averaged relative purity is

$$
\bar{o}_{12}^{(1)}=n_{12}^{-1} \sum_{n=1}^{\infty} \int_{0}^{\infty} d t \int_{0}^{t} d t_{1} \int_{t_{1}}^{t} d t_{2} \ldots \int_{t_{n-1}}^{t} d t_{n} D_{n}\left(t_{1}, \ldots, t_{n}, t\right) o_{12}^{(n)}(t)=\frac{1}{2}-\frac{\eta_{1}\left(\eta_{1}+\eta_{2}\right)}{\eta_{2}\left(6-\eta_{1}-\eta_{2}\right)\left(7-\eta_{2}-4 \eta_{1}\right)} .
$$

(2) The last detection before $t$ was made by observer 1 instead of observer 2 , as in the case (1). The description of this second situation is the same as above, except that observers 1 and 2 are interchanged. In particular, the final result for the relative purity reads

$$
\bar{o}_{12}^{(2)}=\frac{1}{2}-\frac{\eta_{2}\left(\eta_{1}+\eta_{2}\right)}{\eta_{1}\left(6-\eta_{1}-\eta_{2}\right)\left(7-\eta_{1}-4 \eta_{2}\right)} .
$$

In general $\eta_{1} \neq \eta_{2}$ and the two situations are not equally likely. Let us call $p^{(1)}$ the probability that case (1) happens; clearly for case (2) we have $p^{(2)}=1-p^{(1)}$. The probability $p^{(1)}$ is given by $p^{(1)}=n_{12} / n_{1}=\eta_{2} /\left(\eta_{1}+\eta_{2}\right)$. The relative purity averaged over the two situations is then

$$
\begin{aligned}
O_{12} & =\frac{\eta_{2}}{\eta_{1}+\eta_{2}} \bar{o}_{12}^{(1)}+\frac{\eta_{1}}{\eta_{1}+\eta_{2}} \bar{o}_{12}^{(2)} \\
& =\frac{1}{2}-\frac{\eta_{1} \eta_{2}\left[6-2\left(\eta_{1}+\eta_{2}\right)\right]}{2\left(6-\eta_{1}-\eta_{2}\right)\left(3-2 \eta_{1}\right)\left(3-2 \eta_{2}\right)} .
\end{aligned}
$$

In figures 3 and 4 we show simulations of the time evolution of the relative purity $O_{12}$ for the case $\eta_{1}=\eta_{2}$ and $\eta_{1} \neq \eta_{2}$.

Note that the average relative purity is manifestly less than $1 / 2$ : the single observer states $\rho_{1}$ and $\rho_{2}$ are anticorrelated. The reason for this anti-correlation can be explained as follows. Suppose that the states $\rho_{1}, \rho_{2}, \rho$ are initially fully correlated (i.e., relative purity equal to one). Observer 1 is most likely to have a detection when the 


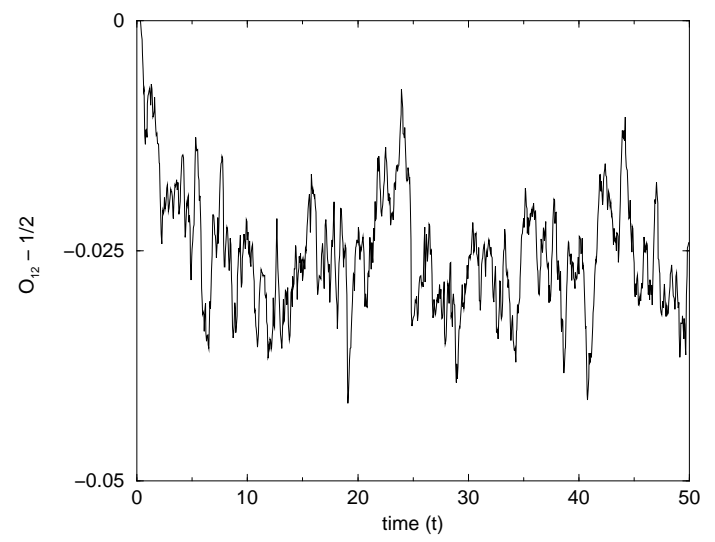

FIG. 3: Average relative purity $O_{12}-1 / 2$ between two observers performing photo-detection measurements. Their initial condition is maximal lack of knowledge, i.e. $\rho_{1}(t=0)=\rho_{1}(t=0)=I / 2$. The efficiencies are $\eta_{1}=\eta_{2}=0.5$. According to Eq. (59) the asymptotic value of the relative purity is -0.025 . The stochastic trajectory is an average over 256 single realizations.

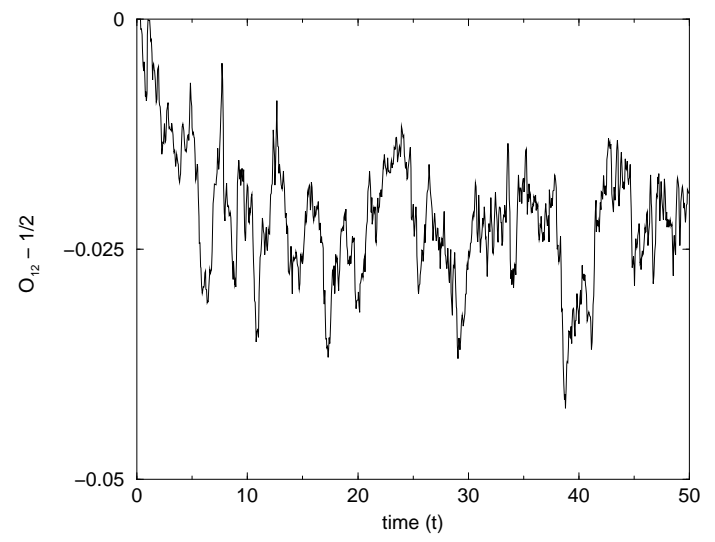

FIG. 4: Average relative purity $O_{12}-1 / 2$ between two observers performing photo-detection measurements. Their initial condition is maximal lack of knowledge, i.e. $\rho_{1}(t=0)=\rho_{1}(t=0)=I / 2$. The efficiencies are $\eta_{1}=0.7$ and $\eta_{2}=0.3$. According to Eq. (59) the asymptotic value of the relative purity is -0.022 . The stochastic trajectory is an average over 256 single realizations.

super-observer's state is excited $(z \approx+1)$. The hypothetical positive correlation means that when $z \approx+1$, then also $z_{1} \approx+1$ and $z_{2} \approx+1$. Suppose that a detection by observer 1 happens. The super-observer $z$ and the single observer $z_{1}$ jump down to -1 . The observer 2 has no clue that there was a detection by observer 1 . What is more, the super-observer $z$ is close to -1 so observer 2 cannot detect a photon and jump to $z_{2}=-1$. His $z_{2}$ remains close to +1 . Just after the detection the product $z z_{1}>0$ but the product $z_{1} z_{2}<0$. This mechanism cannot make $O_{1}<1 / 2$ but it can and it does make $O_{12}<1 / 2$.

We have solved exactly the problem of correlations between multiple measurement channels in the limit of $\omega \gg 1$. This limit is sufficient to illustrate our ideas. However, the exact solution for arbitrary $\omega$ of the resonance fluorescence problem in Ref. [26] suggests that, with some extra work, our formulas for average relative purities can be generalized exactly to arbitrary $\omega$.

\section{TWO-LEVEL ATOM: HOMODYNE DETECTION}

As we saw in the previous section, direct photo-detection is a way to find out if the atom is in the ground state. One can also measure different quadratures of the two-level atom by performing homodyne detection on the radiation emitted from it [18]. In general, it is possible to measure the expectation value of the operator $(x \cos \phi-y \sin \phi)$, where $\phi$ is the phase of the local oscillator in the homodyne detector. This kind of measurement tends to localize the state of the atom around the eigenstates of the operator $\left(\sigma_{x} \cos \phi-\sigma_{y} \sin \phi\right)$. The MCSME is (see Appendix A) 


$$
\begin{aligned}
& d \rho=-i d t\left[\omega \sigma_{x}, \rho\right]+d t\left(c \rho c^{\dagger}-\frac{1}{2} c^{\dagger} c \rho-\frac{1}{2} \rho c^{\dagger} c\right)+\sum_{i}\left(d N_{i}-\overline{d N_{i}(\rho)}\right)\left(\frac{\left(c+\gamma_{i}\right) \rho\left(c^{\dagger}+\gamma_{i}^{\star}\right)}{\operatorname{Tr}\left[\left(c+\gamma_{i}\right) \rho\left(c^{\dagger}+\gamma_{i}^{\star}\right)\right]}-\rho\right), \\
& d \rho_{i}=-i d t\left[\omega \sigma_{x}, \rho_{i}\right]+d t\left(c \rho_{i} c^{\dagger}-\frac{1}{2} c^{\dagger} c \rho_{i}-\frac{1}{2} \rho_{i} c^{\dagger} c\right)+\left(d N_{i}-\overline{d N_{i}\left(\rho_{i}\right)}\right)\left(\frac{\left(c+\gamma_{i}\right) \rho_{i}\left(c^{\dagger}+\gamma_{i}^{\star}\right)}{\operatorname{Tr}\left[\left(c+\gamma_{i}\right) \rho_{i}\left(c^{\dagger}+\gamma_{i}^{\star}\right)\right]}-\rho_{i}\right), \\
& \overline{d N_{i}(\rho)}=\eta_{i} d t\left[R_{i}^{2}+R_{i} e^{-i \phi_{i}} \operatorname{Tr} \rho c+R_{i} e^{+i \phi_{i}} \operatorname{Tr} \rho c^{\dagger}+\operatorname{Tr} \rho c^{\dagger} c\right] .
\end{aligned}
$$

Here $\gamma_{i}=R_{i} \exp \left(i \phi_{i}\right)$ is the complex amplitude of the local oscillator of the detector $i$. We will eventually take the limit $R_{i} \rightarrow \infty$. We allow each observer to have his own homodyne phase $\phi_{i}$, so that they can measure different quadratures, i.e., they measure non-commuting observables (a related experimental realization of measurements of non-commuting observables in two channels in cavity QED was carried out in 25]). The detector currents are proportional to Eq. (62). The case $\phi_{i}=0$ corresponds to measurement of the $x$-quadrature and $\phi_{i}=\pi / 2$ to $y$-quadrature. The large $R_{i}$ limit of Eq. (60) is

$$
\begin{aligned}
d \rho= & -i d t\left[\omega \sigma_{x}, \rho\right]+d t\left(c \rho c^{\dagger}-\frac{1}{2} c^{\dagger} c \rho-\frac{1}{2} \rho c^{\dagger} c\right) \\
& +\sum_{i} \sqrt{\eta_{i}} d W_{i}\left[c \rho e^{-i \phi_{i}}+\rho c^{\dagger} e^{+i \phi_{i}}-\rho \operatorname{Tr}\left(c \rho e^{-i \phi_{i}}+\rho c^{\dagger} e^{+i \phi_{i}}\right)\right],
\end{aligned}
$$

where $d W_{i}$ are gaussian Wiener increments such that $\overline{d W_{i}}=0$ and $\overline{d W_{i} d W_{j}}=\delta_{i j} d t$. To derive the large $R_{i}$ limit of Eq. (61) we first split $d N_{i}-\overline{d N_{i}\left(\rho_{i}\right)}=\left(d N_{i}-\overline{d N_{i}(\rho)}\right)+\left(\overline{d N_{i}(\rho)}-\overline{d N_{i}\left(\rho_{i}\right)}\right)$. In the large $R_{i}$ limit the first term is proportional to $R_{i} \sqrt{\eta_{i}} d W_{i}$, and the second term is proportional to $R_{i} \eta_{i} d t \operatorname{Tr}\left[c\left(\rho-\rho_{i}\right) e^{-i \phi_{i}}+\left(\rho-\rho_{i}\right) c^{\dagger} e^{+i \phi_{i}}\right]$. The large $R_{i}$ limit of Eq. (61) reads

$$
\begin{aligned}
d \rho_{i}= & -i d t\left[\omega \sigma_{x}, \rho_{i}\right]+d t\left(c \rho_{i} c^{\dagger}-\frac{1}{2} c^{\dagger} c \rho_{i}-\frac{1}{2} \rho_{i} c^{\dagger} c\right) \\
& +\left[\sqrt{\eta_{i}} d W_{i}+\eta_{i} d t \operatorname{Tr}\left(c\left(\rho-\rho_{i}\right) e^{-i \phi_{i}}+\left(\rho-\rho_{i}\right) c^{\dagger} e^{+i \phi_{i}}\right)\right]\left[\left(c \rho_{i} e^{-i \phi_{i}}+\rho_{i} c^{\dagger} e^{+i \phi_{i}}\right)-\rho \operatorname{Tr}\left(c \rho_{i} e^{-i \phi_{i}}+\rho_{i} c^{\dagger} e^{+i \phi_{i}}\right)\right] .
\end{aligned}
$$

\section{A. Average relative purity between $\rho_{i}$ and $\rho$}

Unfortunately it is not possible to find analytic solutions to the above equations for all values of the efficiencies $\eta_{i}$. For small values of these efficiencies it is possible to work out various relative purities by a perturbative expansion in powers of $\eta_{i}$. For $\eta_{i}=0$, the conditional master equation (Eq. (63)) is the unconditional master equation, which has a stationary solution $\rho_{s s}$. In the limit $\omega \gg 1$ it is equal to $\rho_{s s}=I / 2$ or $x_{s s}=y_{s s}=z_{s s}=0$. The full density matrix is perturbed from this stationary state by the noises $d W_{i}$, and the magnitude of the perturbation grows with $\eta_{i}$. We expand $\rho=\rho_{s s}+\delta \rho$, the last term containing those perturbations. Let us write $\delta \rho=\left(x \sigma_{x}+y \sigma_{y}+z \sigma_{z}\right) / 2$. We expand $x$ as $x=x^{(1)}+x^{(2)}+\ldots$, where $x^{(1)}$ is of order $\eta_{i}^{1 / 2}, x^{(2)}$ is of order $\eta_{i}^{3 / 2}$, etc. Similar expansions are used for $y$ and $z$. To first order in $\eta_{i}^{1 / 2}$ Eq. (63) reads

$$
\begin{aligned}
& \frac{d x^{(1)}}{d t}=-\frac{1}{2} x^{(1)}+\sum_{i} \sqrt{\eta_{i}} \theta_{i} \cos \phi_{i}, \\
& \frac{d y^{(1)}}{d t}=-\frac{1}{2} y^{(1)}-2 \omega z^{(1)}-\sum_{i} \sqrt{\eta_{i}} \theta_{i} \sin \phi_{i}, \\
& \frac{d z^{(1)}}{d t}=-z^{(1)}+2 \omega y^{(1)} .
\end{aligned}
$$

These equations have a solution

$$
\begin{aligned}
x^{(1)}(t) & =\sum_{i} x_{i}^{(1)}(t), \\
y^{(1)}(t) \pm i z^{(1)}(t) & =\sum_{i}\left[y_{i}^{(1)}(t) \pm i z_{i}^{(1)}(t)\right],
\end{aligned}
$$




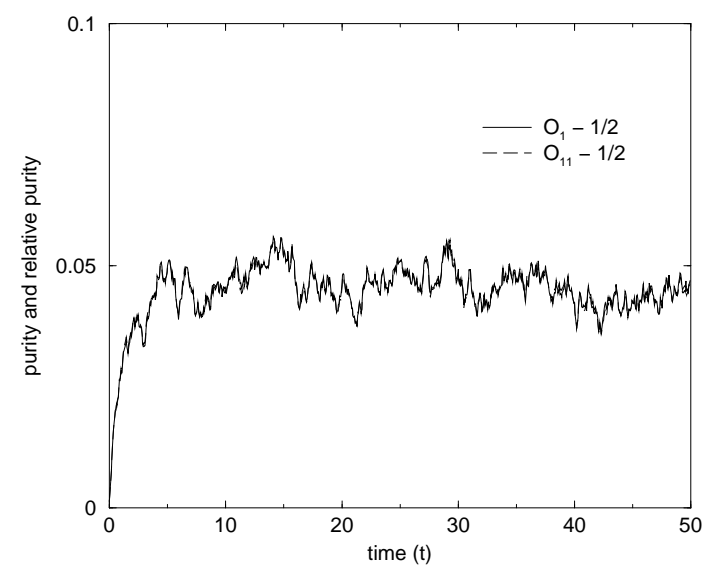

FIG. 5: Average purity $O_{11}-1 / 2$ and average relative purity $O_{1}-1 / 2$ for an observer performing homodyne measurements. The efficiency is $\eta_{1}=0.1$ and the homodyne phase is $\phi_{1}=0$. According to Eq. (70), which is valid for small efficiencies, the asymptotic value of the average relative purity and average purity is $O_{1}-1 / 2=O_{11}-1 / 2=0.05$. In the scale of the figure $O_{1}$ and $O_{11}$ practically coincide. The stochastic trajectories are an average over 256 single realizations.

where

$$
\begin{aligned}
x_{i}^{(1)}(t) & =\sqrt{\eta_{i}} \cos \phi_{i} \int_{-\infty}^{t} d \tau e^{-\frac{1}{2}(t-\tau)} \theta_{i}(\tau), \\
y_{i}^{(1)}(t) \pm i z_{i}^{(1)}(t) & =-\sqrt{\eta_{i}} \sin \phi_{i} \int_{-\infty}^{t} d \tau e^{-\left(\frac{3}{4} \mp 2 i \omega\right)(t-\tau)} \theta_{i}(\tau) .
\end{aligned}
$$

To leading order in $\eta_{i}^{1 / 2}$, s the single observer equation (61) is

$$
\begin{aligned}
\frac{d x_{i}^{(1)}}{d t} & =-\frac{1}{2} x_{i}^{(1)}+\sqrt{\eta_{i}} \theta_{i} \cos \phi_{i}, \\
\frac{d y_{i}^{(1)}}{d t} & =-\frac{1}{2} y_{i}^{(1)}-2 \omega z_{i}^{(1)}-\sqrt{\eta_{i}} \theta_{i} \sin \phi_{i}, \\
\frac{d z_{i}^{(1)}}{d t} & =-z_{i}^{(1)}+2 \omega y_{i}^{(1)} .
\end{aligned}
$$

These equations are solved by the already introduced $x_{i}^{(1)}, y_{i}^{(1)}, z_{i}^{(1)}$. To leading order in $\eta_{i}$ 's the relative purity $O_{i}=\overline{\operatorname{Tr} \rho_{i} \rho}$ is

$$
O_{i} \equiv \frac{1}{2}+\frac{1}{2} \overline{\left[x^{(1)} x_{i}^{(1)}+y^{(1)} y_{i}^{(1)}+z^{(1)} z_{i}^{(1)}\right]}=\frac{1}{2}+\frac{1}{2} \overline{\left[x_{i}^{(1)} x_{i}^{(1)}+y_{i}^{(1)} y_{i}^{(1)}+z_{i}^{(1)} z_{i}^{(1)}\right]} .
$$

A straightforward calculation leads to the following stationary average relative purity

$$
O_{i}=\frac{1}{2}+\eta_{i}\left[\frac{1}{2} \cos ^{2} \phi_{i}+\frac{1}{3} \sin ^{2} \phi_{i}\right] .
$$

As we can see from Eq. (69) the average relative purity coincides with the average purity $O_{i i}$. The latter is the highest for measurement basis correlated to the pointer states basis of the system, i.e., when $\phi_{i}=0$. Through this measurement one can find out most about the system. In figures 5 and 6 we plot the average relative purity $O_{1}$ and the average purity $O_{11}$ for different values of the efficiencies and homodyne phases.

\section{B. Average relative purity $O_{i j}$}

The average relative purity $O_{12}=\overline{\operatorname{Tr} \rho_{1} \rho_{2}}$ is zero to leading order in $\eta_{i}$. To get a nonzero average relative purity we have to go one step further in the perturbative expansion for $x, y$, and $z$. The equations for the second order terms 


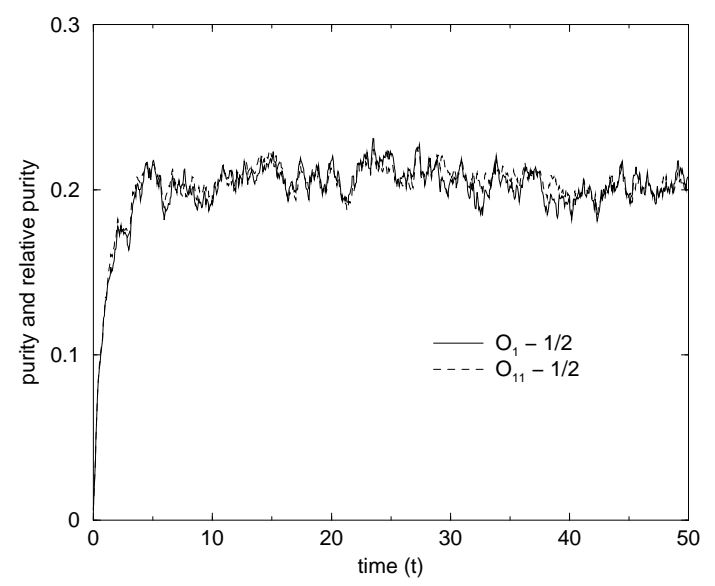

FIG. 6: Average purity $O_{11}-1 / 2$ and average relative purity $O_{1}-1 / 2$ for an observer performing homodyne measurements. The efficiency is $\eta_{1}=0.5$ and the homodyne phase is $\phi_{1}=0$. We do not expect Eq. (70) to hold for such a big efficiency. The stochastic trajectories are an average over 256 single realizations. The small discrepancy between $O_{1}$ and $O_{11}$ in the figure is an artifact of the finite number of trajectories used to calculate the averages.

that follow from the single observer equation Eq. (64) are

$$
\begin{aligned}
\frac{d x_{i}^{(2)}}{d t} & =-\frac{1}{2} x_{i}^{(2)}+\eta_{i} \cos ^{2} \phi_{i} x_{j}^{(1)}-\eta_{i} \sin \phi_{i} \cos \phi_{i} y_{j}^{(1)}, \\
\frac{d y_{i}^{(2)}}{d t} & =-\frac{1}{2} y_{i}^{(2)}-2 \omega z_{i}^{(2)}+\eta_{i} \sin ^{2} \phi_{i} y_{j}^{(1)}-\eta_{i} \sin \phi_{i} \cos \phi_{i} x_{j}^{(1)}, \\
\frac{d z_{i}^{(2)}}{d t} & =-z_{i}^{(2)}+2 \omega y_{i}^{(2)} .
\end{aligned}
$$

Formal solutions of these equations are

$$
\begin{aligned}
x_{i}^{(2)}(t) & =\eta_{i} \int_{-\infty}^{t} d \tau e^{-\frac{1}{2}(t-\tau)}\left(\cos ^{2} \phi_{i} x_{j}^{(1)}(\tau)-\sin \phi_{i} \cos \phi_{i} y_{j}^{(1)}(\tau)\right), \\
y_{i}^{(2)}(t) \pm i z_{i}^{(2)}(t) & =\eta_{i} \int_{-\infty}^{t} d \tau e^{-\frac{3}{4}(t-\tau) \pm 2 i \omega(t-\tau)}\left(\sin ^{2} \phi_{i} y_{j}^{(1)}(\tau)-\sin \phi_{i} \cos \phi_{i} x_{j}^{(1)}(\tau)\right) .
\end{aligned}
$$

To the first non-vanishing order in $\eta_{i}$ the average relative purity is

$$
O_{12}=\frac{1}{2}+\frac{1}{2} \overline{\left[x_{1} x_{2}+y_{1} y_{2}+z_{1} z_{2}\right]}=\frac{1}{2}+\frac{1}{2} \overline{\left[x_{1}^{(1)} x_{2}^{(2)}+y_{1}^{(1)} y_{2}^{(2)}+z_{1}^{(1)} z_{2}^{(2)}\right]}+\frac{1}{2} \overline{\left[x_{1}^{(2)} x_{2}^{(1)}+y_{1}^{(2)} y_{2}^{(1)}+z_{1}^{(2)} z_{2}^{(1)}\right]} .
$$

We evaluate this expression in Appendix C. The result is

$$
O_{12}=\frac{1}{2}+\eta_{1} \eta_{2}\left[\cos ^{2} \phi_{1} \cos ^{2} \phi_{2}+\frac{4}{9} \sin ^{2} \phi_{1} \sin ^{2} \phi_{2}\right] .
$$

The average relative purity is maximized when both observers perform $x$-measurements $\left(\phi_{1}=\phi_{2}=0\right)$. We verified this formula by numerical simulations using $\eta_{1}=\eta_{2}=0.01$. Below, in figures 7 and 8 , we plot the average relative purity $O_{12}$ for different sets of homodyne phases and efficiencies $\eta_{1}=\eta_{2}=0.1$. These efficiencies are beyond the range of validity of Eq. (74).

\section{CONCLUDING REMARKS}

Let us summarize the new results contained in this paper. We have studied continuous quantum measurement with several observers and we have demonstrated that it reduces to the "single observer" case. The key problem 


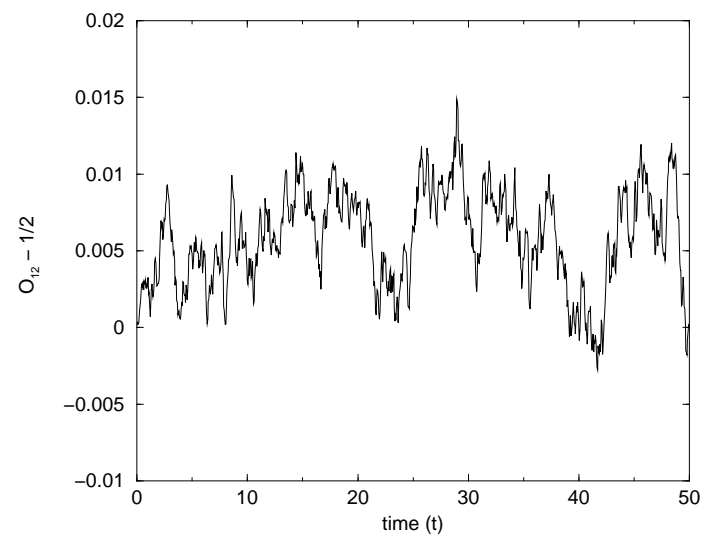

FIG. 7: Average relative purity $O_{12}-1 / 2$ between two observers performing homodyne measurements. The efficiencies are $\eta_{1}=\eta_{2}=0.1$ and the homodyne phases are $\phi_{1}=\phi_{2}=0$. The stochastic trajectory is an average over 256 single realizations.

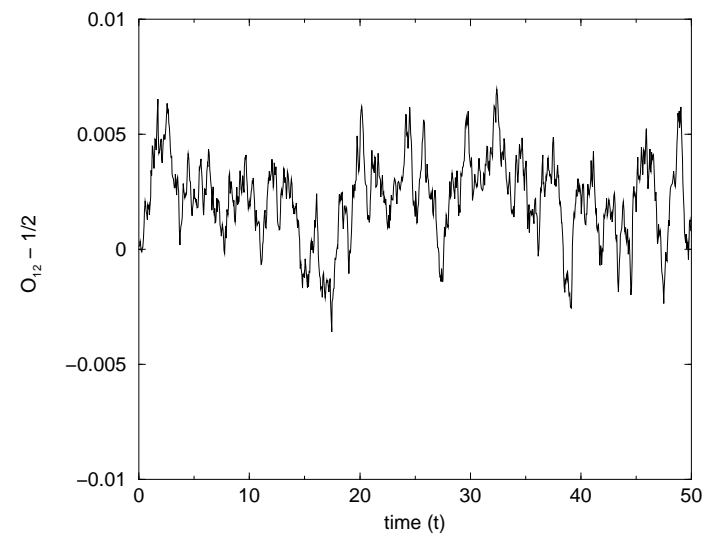

FIG. 8: Average relative purity $O_{12}-1 / 2$ between two observers performing homodyne measurements. The efficiencies are $\eta_{1}=\eta_{2}=0.1$ and the homodyne phases are $\phi_{1}=\phi_{2}=\pi / 2$. The stochastic trajectory is an average over 256 single realizations.

of consistency of the sets of data acquired by different observers is then reduced to the probability that a given combination of data sets will be ever detected by the super-observer. We have applied the formalism to several examples of quantum optics as well as to quantum Brownian motion. Observers gain information about the state of the system from their measurement records. We have shown that observers gain most information about the system and they agree the most when they measure in environmental basis most correlated to the pointer basis of the system.

Several questions regarding correlations between measurement records of different observers were posed. We have shown that the problem of consistency of sets of data acquired by different observers is reduced to the probability that a given combination of data sets will ever be detected by the super-observer. We have introduced average relative purity to study correlations between measurement records of different observers. For the model of zero temperature quantum Brownian motion (which is equivalent to the model of a damped harmonic oscillator) coherent states are perfect pointer states: The solution to Eq. (25) for an initial coherent state remains pure and it is just a coherent state with decaying amplitude. We have shown that for an initial Schrödinger cat state $(|z\rangle+|-z\rangle) / \sqrt{2}$ made of large amplitude coherent states, records of different observers performing measurements on the environment in a basis correlated with the pointer basis will eventually fully agree (as shown in Figure 1), and the average relative purity will be equal to one. For the case when the most predictable states exist, but are not very predictable and are not imprinted on the environment (and, in particular, do not commute with the interaction Hamiltonian), such as the model of two-level atom resonance fluorescence, the agreement between observers' guess of the state of the system may only be partial, and it is even possible to obtain anti-correlation between measurement records, as in the case of photo-detection. 


\section{ACKNOWLEDGMENTS}

We are grateful to Robin Blume-Kohout, Howard Carmichael, Kurt Jacobs, Harold Ollivier, Juan Pablo Paz and Howard Wiseman for discussions. This research was supported in part by NSA. Moreover J.D. was supported in part by a KBN grant 2 P03B 09223.

\section{APPENDIX A: DERIVATION OF THE TWO-LEVEL ATOM PHOTO- AND HOMODYNE DETECTION MASTER EQUATIONS FOR MULTIPLE MEASUREMENT CHANNELS}

Let us assume a two-level atom that interacts with the electromagnetic field, which we shall consider as the environment. We will split this environment into different parts $i$, each of which has associated a detector $i$. For example, $i$ may denote different photon wave vectors. In the rotating wave approximation, the dipole interaction between the atom and the electromagnetic field is

$$
V(t)=i \sum_{i}\left(b_{i}^{\dagger} c-c^{\dagger} b_{i}\right)
$$

where $b_{i}$ and $c$ are annihilation operators for photons and the atom, respectively. At every instant of time $t$, a new part of the environment is interacting with the system. Indeed, a localized photon wave packet arrives at the atom, interacts with it, and then flies away. Subsequently, a new wave packet performs the same process. Imagine that at a given instant of time $t$ the combined state of the atom and the field is $R(t)=\rho(t) \otimes \mu$, where $\rho$ is the density matrix for the atom and $\mu$ is that for the field, which we asumme to be in vacuum, $\mu=\otimes_{i}{ }_{i}|0\rangle\left\langle\left. 0\right|_{i}\right.$. This series of idealizations are called the quantum Markov approximation.

The evolution operator for a time interval $d t$ is $U(t, t+d t)=\exp \left[\sum_{i}\left(d B_{i}^{\dagger} c-c^{\dagger} d B_{i}\right)\right]$, where $d B_{i}(t)=b_{i}(t) d t$ has commutation relations

$$
\left[d B_{i}(t), d B_{j}^{\dagger}(t)\right]=\delta_{i j} d t
$$

that follow from the (singular) commutation relations $\left[d b_{i}(t), d b_{j}^{\dagger}\left(t^{\prime}\right)\right]=\delta_{i j} \delta\left(t-t^{\prime}\right)$. The above commutation relation is of order $d t$ instead of $d t^{2}$ [] , as one might have naively expected. For this reason an expansion to first order in $d t$ of the evolution operator requires a second order expansion in terms of $d B_{i}$ and $d B_{i}^{\dagger}$. When ones discards all the information contained in the environment (which is then traced out) one gets an unconditional master equation for the system

$$
d \rho=-i d t\left[\omega \sigma_{x}, \rho\right]+d t \sum_{i}\left(c \rho c^{\dagger}-\frac{1}{2} c^{\dagger} c \rho-\frac{1}{2} \rho c^{\dagger} c\right) .
$$

The sum over $i$ just re-scales the spontaneous emission rate of the atom. In the following we shall absorb that rescaling in a redefinition of time and set the spontaneous emission rate to one.

If the measurements on the environment are not ignored but kept, the evolution of the system is conditioned upon them. In the case of photo-detection, for most of the time intervals no photons are detected. In this case of null results the density matrix of the system evolves according to

$$
\begin{aligned}
d \rho_{\text {zero }}(t) & =O_{\text {zero }} \rho(t)-\rho(t) \operatorname{Tr}\left(O_{\text {zero }} \rho(t)\right), \\
O_{\text {zero }} \rho(t) & =d t\left(-i[H, \rho]-\frac{1}{2} \sum_{i}\left\{c^{\dagger} c, \rho\right\}\right),
\end{aligned}
$$

which is so constructed as to conserve the trace of $\rho$ under the time evolution. When a photon is measured by any of the detectors, the system discontinuously jumps to the ground state of the atom

$$
\begin{aligned}
d \rho_{\text {one }}(t) & =\sum_{i} d N_{i}\left(\frac{O_{\text {one }} \rho}{\overline{d N_{i}}}-f_{i}\right), \\
O_{\text {one }} \rho(t) & =\eta_{i} c \rho c^{\dagger} d t
\end{aligned}
$$


Here the increments $d N_{i} \in\{0,1\}$ are dichotomic stochastic processes with averages $\overline{d N_{i}(\rho)}=\eta_{i} d t \operatorname{Tr}\left[\rho c^{\dagger} c\right], \eta_{i}$ denotes the fraction of the environment measured by detector $i$, and $f_{i}$ is such that two conditions must be satisfied: 1$)$ when $d \rho=d \rho_{\text {zero }}+d \rho_{\text {one }}$ is averaged over all records $i$, it must reduce to the unconditional master equation, and ;2) $\operatorname{Tr}\left[d \rho_{\text {zero }}+d \rho_{\text {one }}\right]=0$. It then follows that $f_{i}=-\eta_{i} \rho \operatorname{Tr}\left(O_{\text {zero }} \rho\right) / \overline{d N_{i}}$. Finally we get the super-observer master equation for photo-detection

$$
d \rho=-i d t\left[\omega \sigma_{x}, \rho\right]+d t\left(c \rho c^{\dagger}-\frac{1}{2} c^{\dagger} c \rho-\frac{1}{2} \rho c^{\dagger} c\right)+\sum_{i}\left(d N_{i}-\overline{d N_{i}(\rho)}\right)\left(\frac{c \rho c^{\dagger}}{\operatorname{Tr}\left[c \rho c^{\dagger}\right]}-\rho\right) .
$$

The super-observer unconditional master equation (A3) is invariant under the transformation $c \rightarrow c+\gamma_{i}$ and $H \rightarrow H-(i / 2) \sum_{i}\left(\gamma_{i}^{\star} c-\gamma_{i} c^{\dagger}\right)$, where $\gamma_{i}$ is a complex number [7]. This symmetry is helpful for deriving other unravelings of the unconditional master equation, for example the one corresponding to homodyne detection. In this case $\gamma_{i}$ represents the coherent amplitude of the classical field of the local oscillator $i$. Introducing this symmetry into the photo-detector master equation one immediately obtains the homodyne master equation Eq. 60.

\section{APPENDIX B: CALCULATION OF THE DISTRIBUTION OF WAITING TIMES}

In this appendix we calculate the distribution of waiting times $f_{\text {wait }}(\tau)$ for the model of resonance fluorescence from a two-level atom subjected to direct photo-detection. It is given by Eq. (50).

$$
f_{\text {wait }}(\tau) \equiv \sum_{n=0}^{\infty} \int_{0}^{\tau} d t_{1} \int_{t_{1}}^{\tau} d t_{2} \ldots \int_{t_{n-1}}^{\tau} d t_{n} D_{n}\left(t_{1}, \ldots, t_{n}, \tau\right) \frac{\eta_{1}}{2}\left[1+Z\left(\tau-t_{n}\right)\right],
$$

where we recall that

$$
Z\left(\tau-t_{n}\right)=-e^{-\frac{3}{4}(1-\eta)\left(\tau-t_{n}\right)} \cos 2 \omega\left(\tau-t_{n}\right)
$$

and that $D_{n}$ is

$$
D_{n}\left(t_{1}, \ldots, t_{n}, \tau\right)=e^{-\frac{\eta}{2}\left(\tau-t_{n}\right)} \prod_{j=1}^{n} \frac{\eta_{2}}{2} e^{-\frac{\eta}{2}\left(t_{j}-t_{j-1}\right)}\left[1-e^{-\frac{3}{4}(1-\eta)\left(t_{j}-t_{j-1}\right)} \cos 2 \omega\left(t_{j}-t_{j-1}\right)\right],
$$

where $t_{0}=0$ is the time of the last detection by observer 1 . Inserting this equation into the previous one, we see that when doing the $n$ time integrals only two terms will survive: one that stems from the product of all the 1 's in $D_{n}$, and another coming from the products of all the cosines (which will therefore contain factors of the form $\cos ^{2}\left(2 \omega t_{j}\right)$ ). All other terms in the expansion of the product in $D_{n}$ will vanish upon integration. In the $\omega \gg 1$ limit we can replace $\cos ^{2}\left(2 \omega t_{j}\right)$ by $1 / 2$. We then get

$$
\begin{aligned}
f_{\text {wait }}(\tau) & \approx \frac{\eta_{1}}{2} e^{-\frac{\eta \tau}{2}} \sum_{n=0}^{\infty} \int_{0}^{\tau} d t_{1} \ldots \int_{t_{n-1}}^{\tau} d t_{n}\left[\left(\frac{\eta_{2}}{2}\right)^{n}-\left(-\frac{\eta_{2}}{4}\right)^{n} e^{-\frac{3}{4}(1-\eta) \tau} \cos (2 \omega \tau)\right] \\
& =\frac{\eta_{1}}{2} e^{-\frac{\eta \tau}{2}} \sum_{n=0}^{\infty}\left[\frac{1}{n !}\left(\frac{\eta_{2} t}{2}\right)^{n}-\frac{1}{n !}\left(-\frac{\eta_{2} t}{4}\right)^{n} e^{-\frac{3}{4}(1-\eta) \tau} \cos (2 \omega \tau)\right] \\
& =\frac{\eta_{1}}{2} e^{-\frac{\eta_{1}}{2} \tau}+\mathcal{O}\left(\frac{1}{\omega}\right) .
\end{aligned}
$$

\section{APPENDIX C: CALCULATION OF THE RELATIVE PURITY FOR THE TWO-LEVEL ATOM WITH HOMODYNE DETECTION}

In this appendix we derive Eq. (74) for the stationary value of the average relative purity between two measurement channels for the model of resonance fluorescence from a two-level atom subjected to homodyne detection.

We must calculate the different terms of Eq. (73). Using Eq. (72) we have 


$$
\overline{x_{i}^{(1)}(t) x_{j}^{(2)}(t)}=\eta_{j} \int_{-\infty}^{t} d \tau e^{-\frac{1}{2}(t-\tau)}\left(\cos ^{2} \phi_{j} \overline{x_{i}^{(1)}(t) x_{i}^{(1)}(\tau)}-\sin \phi_{j} \cos \phi_{j} \overline{x_{i}^{(1)}(t) y_{i}^{(1)}(\tau)}\right) .
$$

Using Eq. (66) and that $\overline{d W_{i} d W_{j}}=\delta_{i j} d t$, it is easy to show that $\overline{x_{i}^{(1)}(t) y_{i}^{(1)}(\tau)}=\mathcal{O}(1 / \omega)$, so we can discard that term in the previous equation. Also, $\overline{x_{i}^{(1)}(t) x_{i}^{(1)}(\tau)}=\eta_{i} \cos ^{2} \phi_{i} \exp (-(t-\tau) / 2)$. Hence

$$
\overline{x_{i}^{(1)}(t) x_{j}^{(2)}(t)}=\eta_{i} \eta_{j} \cos ^{2} \phi_{i} \cos ^{2} \phi_{j}
$$

Also, $\overline{x_{j}^{(1)}(t) x_{i}^{(2)}(t)}$, which obtains from the interchange $i \leftrightarrow j$, is the same. On the other hand,

$$
\overline{y_{i}^{(1)} y_{j}^{(2)}+z_{i}^{(1)} z_{j}^{(2)}}=\frac{1}{2} \overline{\left(y_{i}^{(1)}+i z_{i}^{(1)}\right)\left(y_{j}^{(2)}-i z_{j}^{(2)}\right)}+\text { h.c. }
$$

To calculate this noise average, we make use of Eqs.6762), and

$$
\begin{aligned}
& \overline{\theta_{i}(\tau) x_{i}^{(1)}\left(\tau^{\prime}\right)}=\sqrt{\eta_{i}} \cos \phi_{i} e^{-\frac{1}{2}\left(\tau-\tau^{\prime}\right)} \theta\left(\tau-\tau^{\prime}\right), \\
& \overline{\theta_{i}(\tau) y_{i}^{(1)}\left(\tau^{\prime}\right)}=-2 \sqrt{\eta_{i}} \sin \phi_{i} e^{-\frac{3}{4}\left(\tau^{\prime}-\tau\right)} \theta\left(\tau-\tau^{\prime}\right) \cos 2 \omega\left(\tau-\tau^{\prime}\right) .
\end{aligned}
$$

where the stochastic noises $\theta_{i}$ are defined as $d W_{i}=\theta_{i} d t$, and $\theta(\tau)$ is the step function. Performing the necessary time integrations and discarding $\mathcal{O}(1 / \omega)$ terms, we get

$$
\overline{y_{i}^{(1)} y_{j}^{(2)}+z_{i}^{(1)} z_{j}^{(2)}}=\frac{4}{9} \eta_{i} \eta_{j} \sin ^{2} \phi_{i} \sin ^{2} \phi_{j}
$$

Finally, the average relative purity between the two single observer density matrices reads

$$
O_{i j}=\frac{1}{2}+\eta_{i} \eta_{j}\left[\cos ^{2} \phi_{i} \cos ^{2} \phi_{j}+\frac{4}{9} \sin ^{2} \phi_{i} \sin ^{2} \phi_{j}\right]
$$

[1] W.H. Zurek, Progress of Theoretical Physics 89, 281 (1993).

[2] W.H. Zurek, Philosophical Transactions of the Royal Society of London Series A-Mathematical, Physical and Engineering Sciences 356, 1793 (1998).

[3] W.H. Zurek, Ann. der Physik (Leipzig) 9, 853 (2000).

[4] W.H. Zurek, Rev. Mod. Phys. 75, 715 (2003).

[5] H.J. Carmichael, An Open Systems Approach to Quantum Optics (Springer, Berlin, 1993).

[6] H.M. Wiseman and G.J. Milburn, Phys. Rev. Lett.70, 548 (1993).

[7] H.M. Wiseman, Ph.D. thesis, University of Queensland, 1994.

[8] A. Barchielli, Int. J. Theor. Phys. 32, 2221 (1993).

[9] W.H.Zurek, Phys. Rev. D 24, 1516 (1981).

[10] W.H. Zurek, Phys. Rev. D 26, 1862 (1982).

[11] D.A.R. Dalvit, J. Dziarmaga, and W.H. Zurek, Phys. Rev. Lett. 86, 373 (2001).

[12] R.E. Peierls, Physics World, 19 (1991).

[13] A version of C. Fuchs' counterexample is given in N.D. Mermin, "Whose knowledge?", in Quantum (Un)speakables: Essays in Commemoration of John S. Bell, eds. R. Bertlmann and A. Zeilinger, Springer Verlag, 2001; quant-ph/0107051

[14] T.D. Brun, J. Finkelstein and N.D. Mermin, Phys. Rev. A 65, 032315 (2002).

[15] V.B. Braginsky and F.Ya. Khalili, Quantum Measurements (Cambridge University Press, Cambridge, 1995).

[16] C.W. Gardiner, Quantum Noise (Springer-Verlag, Berlin, 1991).

[17] R. Jozsa, J. Mod. Opt. 41, 2315 (1994); B.W. Schumacher, Phys. Rev. A 51, 2738 (1995).

[18] See, for example, M.O. Scully and M.S. Zubairy, Quantum Optics (Cambridge University Press, Cambridge, 1999).

[19] W.H. Zurek, S. Habib and J.P. Paz, Phys. Rev. Lett.70, 1187 (1993).

[20] P. Goetsch, R. Graham and F. Haake, Phys. Rev. A 51, 136 (1995). 
[21] P. Goetsch, P. Tombesi and D. Vitali, Phys. Rev. A 54, 4519 (1996).

[22] V. Giovannetti, P. Tombesi and D. Vitali, Phys. Rev. A 60, 1549 (1999).

[23] N.G. van Kampen, Stochastic Processes in Physics and Chemistry (Elsevier, Amsterdam, 1997).

[24] H.M. Wiseman and Z. Brady, Phys. Rev. A 62, 023805 (2000).

[25] G.T. Foster, L.A. Orozco, H.M. Castro-Beltran and H.J. Carmichael, Phys. Rev. Lett. 85, 3149 (2000).

[26] H.J. Carmichael, S. Singh, R. Vyas and P.R. Rice, Phys. Rev. A 39, 1200 (1989).

[27] In Appendix A we derive the MCSME for the model of resonance fluorescence from a two-level atom. From that starting point it is straightforward to get the corresponding master equation for zero temperature quantum Brownian motion.

[28] In the limit $r \gg 1$, the states $|+z\rangle$ and $|-z\rangle$ are approximately orthogonal.

[29] In the interaction picture the nonlinear terms in Eq. (38) average to zero thanks to the fast oscillations with frequency $\omega$. 